SPhT-T03/187

\title{
Renormalization of $\Phi$-derivable approximations in scalar field theories
}

\author{
Jean-Paul Blaizot, Edmond Iancu and Urko Reinosa* \\ Service de Physique Théorique, CEA/DSM/SPhT, \\ 91191 Gif-sur-Yvette Cedex, France.
}

(Dated: October 30, 2018)

\begin{abstract}
We discuss the renormalization of $\Phi$-derivable approximations for scalar field theories. In such approximations, the self-energy is obtained as the solution of a self-consistent equation which effectively resums infinite subsets of diagrams of perturbation theory. We show that a consistent renormalization can be carried out, and we provide an explicit construction of the counterterms needed to eliminate the subdivergences. The counterterms are calculated from the solution of an auxiliary gap equation which determines the leading asymptotic part of the self-energy. This auxiliary gap equation may be chosen as the gap equation of the massless theory at zero temperature. We verify explicitly that the counterterms determined at zero temperature are sufficient to eliminate the divergences which occur in finite temperature calculations.
\end{abstract}

*Electronic address: blaizot@spht.saclay.cea.fr, iancu@spht.saclay.cea.fr, reinosa@hep.itp.tuwien.ac.at 


\section{INTRODUCTION}

Self-consistent approximations, such as the $\Phi$-derivable approximations, offer a way to deal with physical situations where the quasiparticle picture is a good starting point. They allow one to include large effects of the interactions into the propagators of quasiparticles, leaving relatively weak residual interactions among them. Such approximations were introduced many years ago in the context of the non-relativistic many body problem 1, 2, 3], and have been reformulated later in the language of relativistic field theory 4]. They have been used recently in various field theoretical problems. In particular, they form the basis for a quasiparticle description of the equilibrium thermodynamics of the quark-gluon plasma [5], which exploits the remarkable simplifications they lead to in the calculation of the entropy [6] (along similar lines, models inspired by the 2loop $\Phi$-derivable approximation for QCD have also been derived [7]). They constitute a framework for variational approximations [8] and they provide a more general alternative to screened perturbation theory [9]. They have been recently applied to the dynamics of quantum fields out of equilibrium [10]. $\Phi$-derivable approximations are known to be "conserving" approximations [2], that is, they are consistent with global symmetries of the Lagrangian 11]. However since they lead to modifications of the propagators while leaving the vertices unaffected, they violate the Ward identities associated to local symmetries; consequences of such violations have been recently explored quantitatively [12], and shown to be suppressed with respect to naive estimates based on power counting.

One of the main obstacles for implementing such non-perturbative approximations in quantum field theory is the difficulty of their renormalization. The central equation that one has to solve is a self-consistent Dyson equation, or gap equation, for the propagator. Such a gap equation effectively resums an infinite set of Feynman diagrams contributing to the self-energy, with arbitrary powers in the coupling constant. We loose the expansion in powers in the coupling as a tool to organize the divergences of the various diagrams. Indeed, at each order in an expansion of the coupling, only a subset of the diagrams of perturbation theory contributing to this order is effectively taken into account by 
the gap equation. The standard proofs of renormalizability do not therefore immediately guarantee that the divergences can be systematically eliminated. Thus, with the exception of the simplest self-consistent mean field approximations, which can be solved easily (see for instance [13, 14]), the renormalization of $\Phi$-derivable approximations has remained a difficult and unsolved problem.

One of the motivations for studying $\Phi$-derivable approximations is their potential usefulness in quantum field theory at finite temperature. A problem that one meets there is the possible occurence of temperature dependent infinities. While, on general grounds (see for instance [15]), one does not expect the temperature to generate any new infinities, it is not always straightforward to guarantee in practical calculations beyond perturbation theory that potentially divergent terms dependent on the temperature do actually cancel. This is especially the case in situations where the various divergences cannot be classified according to some expansion in a small parameter. (For a simple model calculation where one can verify this cancellation explicitly in the first non trivial order in a $1 / N$ expansion, see [16].) It may happen then that one is led to introduce counterterms which depend on the temperature (see for instance [8]), which obscures the physical interpretation of the results.

A major progress on this issue was achieved recently by van Hees and Knoll in a series of papers [11, 17, 18]. The strategy that they put forward [17] is based on an expansion of the self-consistent propagator around the corresponding propagator in the vacuum. They were able to prove the elimination of the temperature dependent divergences, and have established a well defined scheme to solve the gap equation [11, 18]. However, several features of their approach remain somewhat unsatisfactory. The real time formalism that they use, which appears to be essential at some stages of their derivation, does not allow an easy comparison with more conventional field theoretical techniques. More importantly, by focusing on the temperature-dependent divergences alone, they introduce disymmetrical treatments of the vacuum sector and the finite temperature one. This hides the fact that the particular structure of subdivergences that one needs to deal with to guarantee the elimination of temperature dependent infinities is already present in the 
vacuum. Thus, they do not address the issue of renormalizability in the vacuum sector itself, but take it for granted. Finally, the approach by van Hees and Knoll cannot be applied as it stands to massless theories, since an expansion around the vacuum (massless) propagator would be afflicted with infrared divergences. This spoils a main benefit of the self-consistent approximations, which is to regulate the infrared behaviour via a resummation of the medium effects.

In this paper, we present a general discussion of the renormalization of $\Phi$-derivable approximations for scalar field theories. We do not rely on a separation of the vacuum sector from the finite temperature one, and shall use the imaginary time formalism, making the connection with standard field theory transparent. In fact we carry out the renormalization at zero temperature, and proceed as in ordinary perturbation theory, by analyzing the subdivergences of the diagrams which are generated as one iterates the gap equation. The analysis and elimination of divergences is carried out according to the Bogoliubov-Parasiuk-Hepp (BPH) procedure [15, 19], by first removing subdivergences using previously determined counterterms and then absorbing the remaining divergences into corrections to these counterterms. As we shall see, vertex divergences occur, which are identified with those of a special Bethe-Salpeter equation for the 4-point function. The counterterms will be explicitly constructed, as well as a finite gap equation. At the end of our analyzis, it will become straightforward to show that the temperature-dependent contributions are finite, as expected: potential temperature dependent divergences cancel out as one eliminates the subdivergences. But since carried out already in the vacuum, our proof of the renormalizability of the $\Phi$-derivable approximations is a general one, and applies to any of their potential applications, in particular to off-equilibrium problems.

The outline of the paper is the following. In the next section, we recall the essential features of $\Phi$-derivable approximations for the $\phi^{4}$ scalar field theory, their diagrammatic content and basic definitions concerning their renormalization. In the next two sections, we discuss thoroughly the renormalization of simple approximations based on the 2-loop and 3-loop skeletons, respectively. The discussion is carried out in such a way as to prepare for the generalization presented in the last section. A preliminary account of this 
work was given in 20$]$.

\section{GENERAL FORMALISM}

As a generic example of a scalar field theory, we shall consider that described by the Lagrangian

$$
\mathcal{L}=\frac{1}{2}(\partial \phi)^{2}-\frac{m^{2}}{2} \phi^{2}-\frac{\lambda}{4 !} \phi^{4}
$$

and discuss only the symmetric phase in which the field expectation value vanishes, that is, we shall not treat the case of spontaneously broken symmetries.

\section{A. Skeleton expansion for the thermodynamical potential}

The starting point of $\Phi$-derivable approximations is the following expression for the thermodynamic potential $\Omega$, considered as a functional of the full propagator $D[1,2$, 3$]$ :

$$
\beta \Omega[D]=\frac{1}{2} \operatorname{Tr} \log D^{-1}-\frac{1}{2} \operatorname{Tr} \Pi D+\Phi[D],
$$

where $\operatorname{Tr}$ denotes the trace in configuration space, and $\beta=1 / T$, with $T$ the temperature. The trace over configuration space involves integration over imaginary time and over spatial coordinates. Alternatively, these can be turned into summations over Matsubara frequencies and integrations over spatial momenta [21, 22]:

$$
\int_{0}^{\beta} \mathrm{d} \tau \int \mathrm{d}^{3} x \rightarrow \beta V \int[\mathrm{d} k],
$$

where $V$ is the spatial volume, $K^{\mu}=\left(i \omega_{n}, \mathbf{k}\right)$ and $\omega_{n}=n \pi T$, with $n$ an even integer. We have introduced a condensed notation for the measure of the loop integrals (i.e., the sum over the Matsubara frequencies $\omega_{n}$ and the integral over the spatial momentum $\mathbf{k}$ ):

$$
\int[\mathrm{d} k] \equiv T \sum_{n, \text { even }} \int \frac{\mathrm{d}^{3} k}{(2 \pi)^{3}} .
$$


At zero temperature, $\beta \rightarrow \infty$, the loop integrals become ordinary Euclidean integrals in four dimensions:

$$
\int[\mathrm{d} k] \longrightarrow \int \frac{\mathrm{d}^{4} k}{(2 \pi)^{4}},
$$

and $\Omega$ becomes the ground state energy. In the following we shall often use the shorthand notation $\int_{K}$ to denote either the sum integral (II.4) at finite temperature, or the Euclidean integral (II.5), depending upon the context.

The sum-integrals in equations like Eq. (II.2) contain ultraviolet divergences and require regularization. Unless explicitly stated otherwise, we shall assume throughout dimensional regularization.

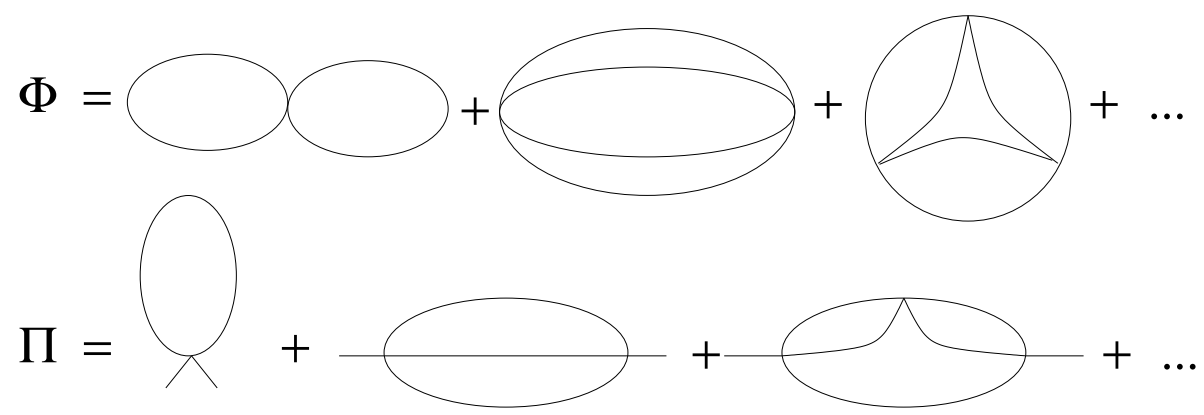

FIG. 1: The skeleton diagrams contributing to the thermodynamical potential to order four loops in scalar $\phi^{4}$ theory, and, below, the corresponding contributions to the self energy to order three loops.

The self-energy $\Pi$ in Eq. (II.2) is related to $D$ by Dyson's equation

$$
D^{-1}=D_{0}^{-1}+\Pi,
$$

where $D_{0}$ denotes the free propagator.

The quantity $\Phi[D]$ is the sum of the 2-particle-irreducible "skeleton" diagrams, i.e., diagrams that cannot be separated into two disconnected parts by cutting two lines. In short, skeletons are diagrams of perturbation theory in which one cannot identify selfenergy insertions. The skeletons contributing to the thermodynamic potential up to four loops and, respectively, to the self-energy up to three loops, are displayed in Fig. 1 In 
Eq. (II.2), $\Phi[D]$ is calculated by associating full propagators $D$ to the lines of the skeletons (while skeleton diagrams in perturbation theory would be calculated with free propagators $\left.D_{0}\right)$.

There are several ways to obtain Eq. (II.2): by integration over the coupling constant 1], by a Legendre transform with respect to a bilocal source [3, 4], or via a simple diagrammatic analysis [3]. We briefly recall here a derivation based on the latter approach. This will give us the oportunity to introduce concepts that will be useful later on.

Consider then the self-energy П. From its definition in Eq. (II.6), it is the sum of all the one-line-irreducible diagrams contributing to the propagator. Clearly, the self-energy skeletons can be obtained from the skeletons of $\Omega$ by cutting one line of the latter (see Fig. 1 for examples). Thus we have:

$$
\frac{\delta \Phi\left[D_{0}\right]}{\delta D_{0}}=\frac{1}{2} \Pi_{s}\left[D_{0}\right],
$$

where $\Pi_{s}\left[D_{0}\right]$ denotes the sum of self-energy skeletons evaluated with free propagators. Now, it is easy to verify that all the diagrams of perturbation theory contributing to $\Pi$ can be obtained from the self-energy skeletons by replacing their internal lines by full propagators. In other words:

$$
\Pi=\Pi_{s}[D]=2 \frac{\delta \Phi[D]}{\delta D} .
$$

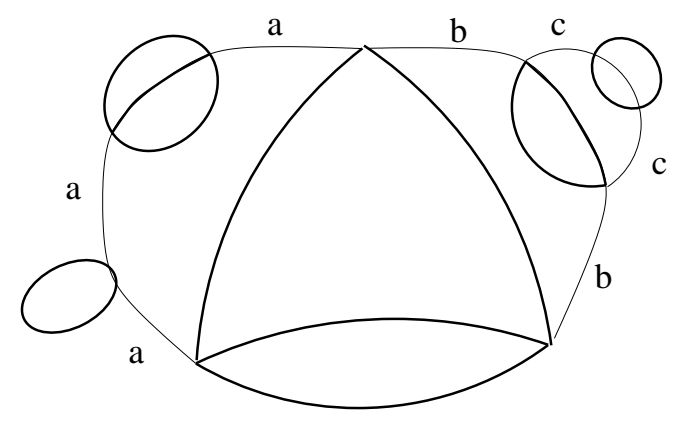

FIG. 2: A typical diagram contributing to the thermodynamical potential

This simple procedure does not hold, however, for the diagrams contributing to the thermodynamical potential because, in the absence of external lines, the diagrams obtained by replacing the free propagators in a skeleton by full propagators are overcounted. 
Consider, as an example, the diagram in Fig. 2. One can identify on it five $\Phi$-skeletons (by replacing successively by propagators the parts which can be regarded as self-energy insertions); these skeletons belong to the set drawn in the first line of Fig. 1 The lines labelled $a, b$, and $c$ in Fig. 22 are said to belong to cycles (there are three cycles in Fig. (2). The cycles have the following property: By cutting two lines of a cycle, one cuts the diagram into two disconnected parts. Cutting two lines belonging to two different cycles leaves the diagram connected. Clearly, two cycles of lines have in common at most one $\Phi$ skeleton (identified by replacing each cycle attached to it by a propagator), but a skeleton may have several cycles attached to it. In other words the cycles generate a tree structure, whose branches are chains of skeletons attached by cycles of lines (an illustration of such a structure is given in Fig. 固 below). This particular structure underlies the algebraic derivation of Eq. (II.2) in terms of a Legendre transform with respect to a bilocal source 3, 4].

For a given diagram $\gamma$, let $n_{c}$ be the number of cycles, $n_{s}$ the number of $\Phi$-skeletons and $n_{l}$ the number of lines belonging to cycles. The following identity is easily proved by induction:

$$
n_{c}+n_{s}-n_{l}=1 .
$$

For the diagram in Fig. 2] we have $n_{c}=3, n_{s}=5, n_{l}=7$. Let now $w(\gamma)$ be the contribution of the diagramm $\gamma$, calculated with the rules of perturbation theory (i.e., with free propagators). We have the obvious identity:

$$
\sum_{\gamma} w(\gamma)=\sum_{\gamma}\left[n_{c}(\gamma)+n_{s}(\gamma)-n_{l}(\gamma)\right] w(\gamma) .
$$

The usefulness of this relation is that the three terms in its right hand side correspond to the three terms in Eq. (II.2), as we now show 23].

We have :

$$
\Phi[D]=\sum_{\gamma} n_{s}(\gamma) w(\gamma)
$$

This states simply that when one evaluates the skeletons with full propagators one counts each diagram $\gamma$ as many times as one can identify skeletons in $\gamma$. For instance the diagram 
of Fig. 2 will occur once in the 1-loop skeleton of Fig. 1 three times in the 2-loop skeleton, and once in the 3-loop skeleton.
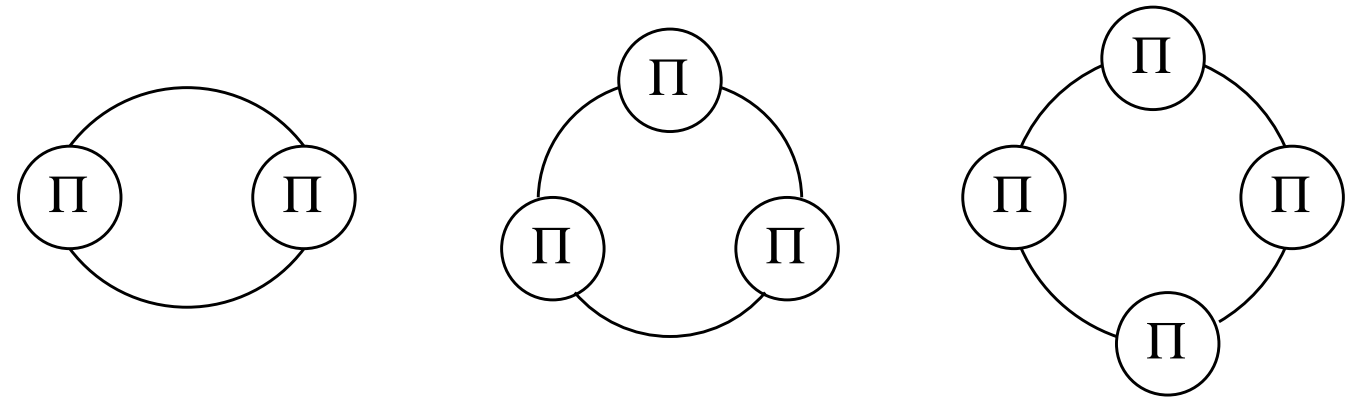

FIG. 3: Ring diagrams, whose contribution is given in Eq. (II.12)

Consider now the sum of the family of diagrams of Fig. 3 corresponding to a cycle of lines with an arbitrary number $n(n \geq 2)$ of self-energy insertions. This is given by

$$
\frac{1}{2} \operatorname{Tr} \log \left(1+D_{0} \Pi\right)-\frac{1}{2} \operatorname{Tr} D_{0} \Pi=\sum_{\gamma} n_{c}(\gamma) w(\gamma)
$$

Again, the right hand side follows from the fact that the diagram $\gamma$ is counted as many times in the left hand side as there are cycles of lines in $\gamma$.

Finally consider one line of a cycle, and view it as part of a full propagator closed on a self-energy. We have then

$$
-\frac{1}{2} \operatorname{Tr} \Pi D+\frac{1}{2} \operatorname{Tr} \Pi D_{0}=\sum_{\gamma} n_{l}(\gamma) w(\gamma)
$$

since $\gamma$ will be counted as many times in the left hand side as it contains lines of cycles.

By adding together the contributions obtained in Eqs. (II.11), (II.12) and (II.13) we recover Eq. (II.2) up to the term term $(1 / 2) \operatorname{Tr} \log D_{0}^{-1}$ which is proportional to the thermodynamical potential in the absence of interactions. 


\section{B. $\Phi$-derivable approximations}

An important property of the functional $\Omega[D]$ is to be stationary under variations of $D$ (at fixed $D_{0}$ ) around the physical propagator:

$$
\delta \Omega[D] / \delta D=0
$$

It is indeed easily verified that this equation for $D$ is equivalent to the self-consistent Dyson equation (commonly referred to as the gap equation):

$$
D^{-1}=D_{0}^{-1}+\Pi[D], \quad \Pi[D]=2 \frac{\delta \Phi[D]}{\delta D} .
$$

The physical thermodynamical potential is then obtained as the value of $\Omega[D]$ for $D$ solution of this equation.

Self-consistent (or variational) approximations, i.e., approximations which preserve the stationarity property (II.14), are obtained by selecting a class of skeletons in $\Phi[D]$ and calculating $\Pi$ from Eq. (II.8). Such approximations are commonly called " $\Phi$-derivable" 2].

By selecting a skeleton in $\Phi$, one obtains an approximation that effectively resums, through the iteration of the gap equation, an infinite subset of diagrams of perturbation theory. An example of such a resummation is illustrated in Fig. 4 a corresponding to the approximation based on the 3-loop skeleton. One recognizes the topology discussed above for the diagram contributing to $\Omega$ : a tree structure, with skeletons joined together by cycles of lines. Consider now the diagrams obtained by opening one line of $\Pi(P)$ which does not belong to a cycle of lines (thereby selecting one branch in the tree alluded to before). Let $K$ be the momentum flowing through the corresponding propagator $D_{0}$. The resulting diagram is a contribution to a 4-point function that we shall call $\Gamma(P, K)$ (see Fig. 4.b). The diagram may contain cycles with more than two lines, that is, lines carrying self-energy insertions. Let then $\Gamma_{0}$ be the set of diagrams of $\Gamma$ which do not carry such insertions, i.e., each cycle of $\Gamma_{0}$ contains only two lines (see Fig. 4. $\mathrm{c}$ ). The diagrams of $\Gamma_{0}$ are two-line reducible. Let us call $\Lambda_{0}$ the contribution to $\Gamma_{0}$ which is two-line irreducible (cf. Fig. 4. d): $\Lambda_{0}(P, K)$ is the sum of all the diagrams contributing to $\Gamma_{0}(P, K)$ which, 


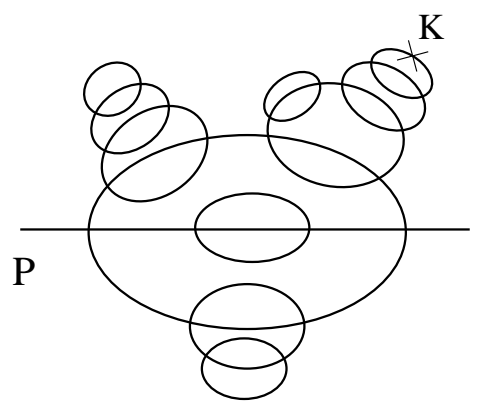

(a)

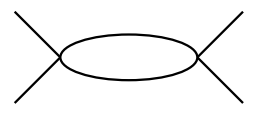

(d)

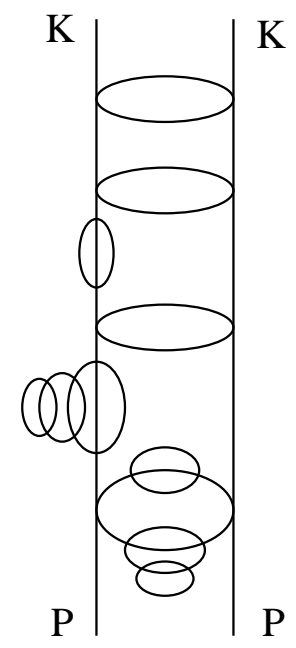

(b)

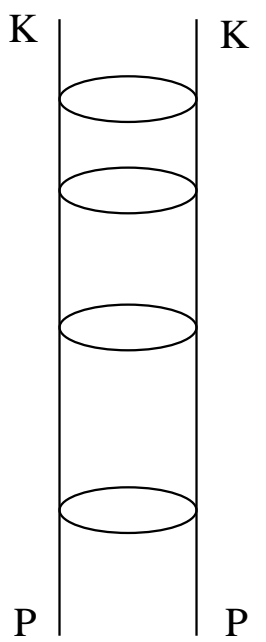

(c)

FIG. 4: (a): A typical diagram contributing to $\Pi$ when the 3-loop skeleton is chosen for $\Phi$. (b): A diagram contributing to $\Gamma(P, K)$, and which is obtained by opening the line marked by a cross in (a). (c): The corresponding contribution to $\Gamma_{0}(P, K)$. (d): The kernel $\Lambda_{0}$ whose iteration produces the diagram $(\mathrm{c})$.

by cutting two internal lines, cannot be split into two pieces, one containing the lines carrying $P$ the other the lines carrying $K$. Clearly, the diagrams contributing to $\Gamma_{0}$ have the form of chains of $\Lambda_{0}$ 's joined together by pairs of propagators $D_{0}$ (see Fig. 4. c). The diagrams that we have identified play the role of skeletons for $\Gamma$ and $\Lambda$, respectively. As was the case for $\Pi$, the presence of external lines makes the identification of skeletons unambiguous, and the complete set of diagrams contributing to $\Gamma$ and $\Lambda$ can be obtained by replacing in $\Gamma_{0}$ and $\Lambda_{0}$ the free propagators $D_{0}$ by the full propagator $D$ (cf. Eq. (II.8) for $\Pi)$.

It it not difficult to show that $\Lambda$ can be obtained from $\Pi$ by functional differentiation:

$$
\Lambda(P, K)=2 \frac{\delta \Pi(P)}{\delta D(K)}=4 \frac{\delta^{2} \Phi}{\delta D(K) \delta D(P)}=\Lambda(K, P)
$$

The contributions to $\Lambda$, up to 2-loop, are displayed in Fig. 5 . As for the diagrams of $\Gamma$, 


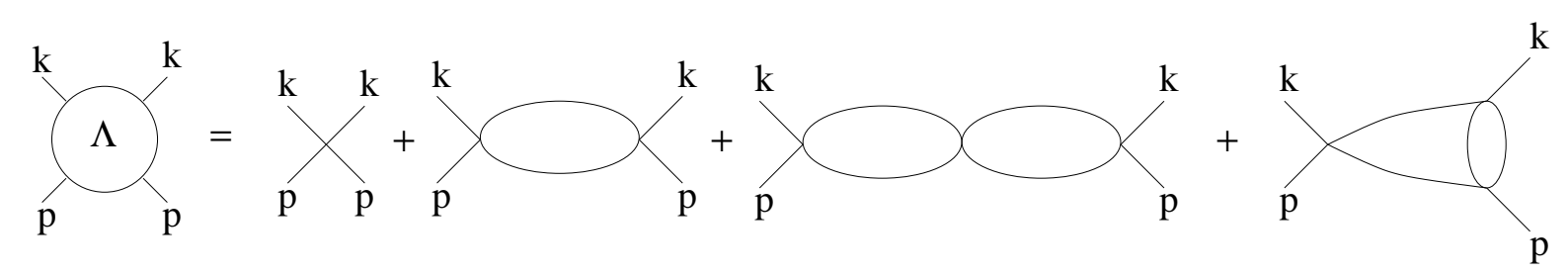

FIG. 5: The skeleton diagrams contributing to the kernel of the Bethe-Salpeter equation up two loops

they are obtained by solving iteratively the following Bethe-Salpeter equation (see Fig. [6):

$$
\begin{aligned}
\Gamma(P, K) & =\Lambda(P, K)-\frac{1}{2} \int_{Q} \Lambda(P, Q) D^{2}(Q) \Gamma(Q, K) \\
& =\Lambda(P, K)-\frac{1}{2} \int_{Q} \Gamma(P, Q) D^{2}(Q) \Lambda(Q, K) .
\end{aligned}
$$

Note that the symmetry property $\Lambda(P, K)=\Lambda(K, P)$ entails the corresponding property for the full 4-point function: $\Gamma(P, K)=\Gamma(K, P)$. This is easily verified by solving Eq. (II.17) iteratively. Note also that the Bethe-Salpeter equation (II.17) realizes a resummation in a specific channel, which one may call the $t$-channel: this is the channel in which the kernel $\Lambda$ is 2-particle irreducible. It follows that as soon as one restricts oneself to a limited class of skeletons, one looses the crossing symmetry of the general 4-point function. This is a general difficulty with $\Phi$-derivable approximations (see also [1]]).

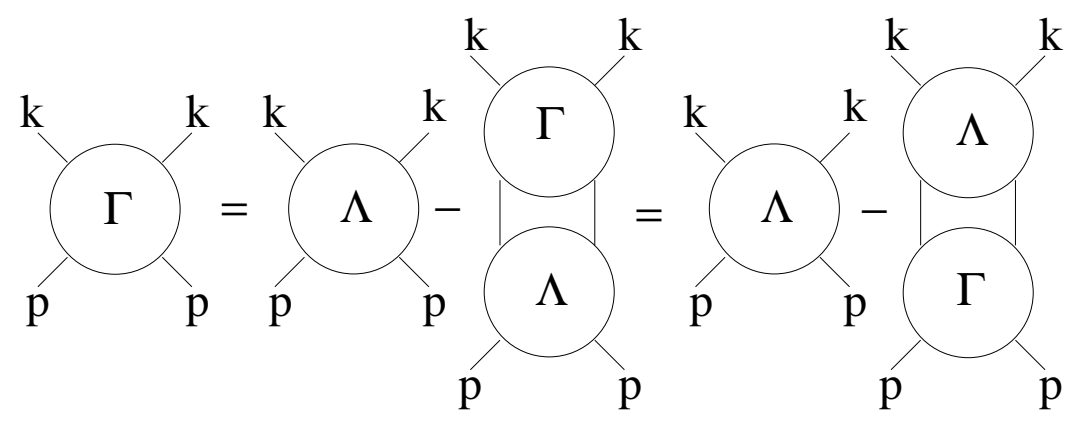

FIG. 6: The Bethe-Salpeter equation in the $t$-channel

Although $\Gamma$ is a function of two momenta while $D$ is a function of a single momentum, the equation for $\Gamma$ is somewhat simpler than the gap equation for $D$. This is because 
the equation for $\Pi$ is a self-consistent equation: $\Pi$ enters the calculation of the dressed propagator which in turns is used to calculate $\Pi$. In contrast, the 4-point function $\Gamma$ does not enter the determination of the kernel $\Lambda$ of the Bethe-Salpeter equation.

For the construction of the mass counterterm, it will be later convenient to consider also the derivative of the self-energy with respect to the mass. In perturbation theory, the mass enters only the free propagator $D_{0}$, and $\partial D_{0} / \partial m^{2}=-D_{0}^{2}$. Thus the diagrams for $m^{2} \partial \Pi / \partial m^{2}$ are those obtained by inserting $m^{2}$ in one of the internal propagators. A simple analysis then shows that

$$
m^{2} \frac{\partial \Pi(P)}{\partial m^{2}}=-\frac{m^{2}}{2} \int_{K} \Gamma(P, K) D^{2}(K) .
$$

This equation can be understood as follows: Consider first those diagrams contributing to $m^{2}\left(\partial \Pi / \partial m^{2}\right)$ which contain no self-energy insertions (skeletons). It is then not hard to see that the contribution of these diagrams is given by the r.h.s. of Eq. (II.18) in which $D$ is replaced by $D_{0}$, and similarly $\Gamma$ is replaced by $\Gamma_{0}$. Eq. (II.18) then follows by noticing that the complete set of diagrams contributing to $m^{2}\left(\partial \Pi / \partial m^{2}\right)$ is obtained by replacing in the skeleton diagrams identified above the free propagators $D_{0}$ by the full ones $D$.

Eq. (II.18) may be also obtained by the following algebraic manipulations that will be

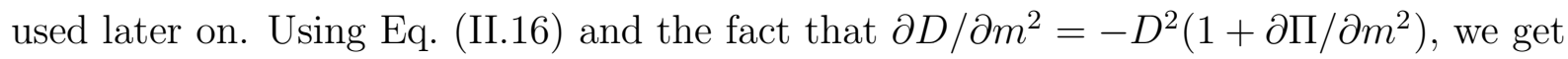

$$
m^{2} \frac{\partial \Pi(K)}{\partial m^{2}}=-\frac{m^{2}}{2} \int_{Q} \Lambda(K, Q) D^{2}(Q)\left(1+\frac{\partial \Pi(Q)}{\partial m^{2}}\right)
$$

which we can rearrange as follows

$$
\int_{Q}\left[\delta(K-Q)+\frac{1}{2} \Lambda(K, Q) D^{2}(Q)\right] \frac{\partial \Pi(Q)}{\partial m^{2}}=-\frac{1}{2} \int_{Q} \Lambda(K, Q) D^{2}(Q) .
$$

Now, the Bethe-Salpeter equation (II.17) can be written as

$$
\int_{Q}\left[\delta(P-Q)+\frac{1}{2} \Lambda(P, Q) D^{2}(Q)\right] \Gamma(Q, K)=\Lambda(P, K),
$$

or equivalently as

$$
\int_{K}\left[\delta(P-K)-\frac{1}{2} \Gamma(P, K) D^{2}(K)\right] \Lambda(K, Q)=\Gamma(P, Q) .
$$


From these, one easily obtains

$$
\int_{K}\left[\delta(P-K)-\frac{1}{2} \Gamma(P, K) D^{2}(K)\right]\left[\delta(K-Q)+\frac{1}{2} \Lambda(K, Q) D^{2}(Q)\right]=\delta(P-Q) .
$$

Using these equations, one easily transforms Eq. (11.20) into Eq. (11.18). Renormalization counterterms will slightly modify the form of this equation, as we shall see shortly.

\section{Renormalization: generalities}

In $\phi^{4}$ theory in four dimensions, we know from power counting that, aside from the thermodynamical potential itself, only the 2-point and the 4-point functions can be divergent when computed in perturbation theory. Thus, one expects the subdivergences in any diagram of perturbation theory to be of two types, associated respectively with divergences of a 2-point and a 4-point function. An important class of 4-point subdivergences in a self-energy diagram will contain as external lines those of the self-energy itself and those obtained by cutting a line (associated with a free propagator) which does not belong to a cycle of lines. The analysis done previously indicates that this is nothing but a contribution to $\Gamma$. One should therefore expect a strong interplay between the vertex subdivergences of the gap equation and those of the Bethe-Salpeter equation.

The divergences of the $n$-point functions can be absorbed in local counterterms corresponding to a redefinition of the parameters of the lagrangian. The initial theory depends

on three parameters, the bare mass and coupling constant, $m_{B}$ and $\lambda_{B}$ respectively, and an ultraviolet cut-off. The relations between the renormalized and the bare parameters (denoted by a subscript $B$ ) are the usual ones:

$$
\phi_{B}=\sqrt{Z} \phi, \quad Z m_{B}^{2}=m^{2}+\delta m^{2}, \quad Z^{2} \lambda_{B}=Z_{\lambda} \lambda=\lambda+\delta \lambda, \quad \delta Z=Z-1 .
$$

The parameters of the renormalized theory are the renormalized mass and coupling constant, $m$ and $\lambda$ respectively, and the counterterms $\delta Z, \delta m^{2}, \delta \lambda$ are supposed to be functions of $m, \lambda$ and the cut-off. They are such that physical observables, expressed in terms of renormalized quantities, have a finite limit when the cut-off goes to infinity. 
The relation between bare and renormalized propagators reads:

$$
D_{B}=Z D
$$

and we define bare and renormalized self-energies by

$$
D_{B}^{-1}(K)=K^{2}+m_{B}^{2}+\Pi_{B} \quad D^{-1}(K)=K^{2}+m^{2}+\Pi .
$$

Thus $\Pi$ and $\Pi_{B}$ are related by

$$
\Pi=Z \Pi_{B}+K^{2} \delta Z+\delta m^{2}
$$

For the skeleton diagrams, we have the identity

$$
\Phi\left[\lambda_{B}, D_{B}\right]=\Phi\left[Z_{\lambda} \lambda, D\right]
$$

This follows from the fact that to each vertex of $\Phi$ are attached four propagators. Thus, in $\Phi$, one can replace $D_{B}=Z D$ by $D$ provided one replaces at the same time $\lambda_{B}$ by $Z^{2} \lambda_{B}=Z_{\lambda} \lambda$. This relation (II.28), together with the definitions above, allows us to express the thermodynamical potential $\Omega$ in terms of renormalized quantities

$$
\beta(\Omega[D]-\Delta \Omega)=\frac{1}{2} \int_{K} \log D^{-1}(K)-\frac{1}{2} \int_{K}\left(\Pi(K)-\delta Z K^{2}-\delta m^{2}\right) D(K)+\Phi\left[Z_{\lambda} \lambda, D\right],
$$

where $\Delta \Omega$ is an infinite constant, independent of the temperature, whose role is to absorb the global divergence which remains in $\Omega$ once the 2-point and 4-point function subdivergences have been properly eliminated. When expressed in terms of bare quantities, the thermodynamical potential is stationary with respect to variations of $D_{B}$, at fixed bare parameters (see Eq. (II.14) ). One can verify that a similar property holds when $\Omega$ is expressed in terms of the renormalized propagator, i.e., the expression (II.29) is stationary with respect to variations of $D$ at fixed renormalized parameters (and therefore fixed values of the counterterms). We have also $\delta \Phi / \delta D_{B}=(1 / 2) \Pi_{B}$, and therefore:

$$
\frac{\delta \Phi\left[Z_{\lambda} \lambda, D\right]}{\delta D}=\frac{1}{2} Z \Pi_{B}
$$


with $\Pi_{B}$ evaluated with the propagator $D$. Given the relation (I.26) between $\Pi_{B}$ and $\Pi$, one then obtains the gap equation in the form:

$$
\Pi(K)=\delta Z K^{2}+\delta m^{2}+2 \frac{\delta \Phi\left[Z_{\lambda} \lambda, D\right]}{\delta D} .
$$

Consider finally the Bethe-Salpeter equation. In terms of bare quantities it reads:

$$
\Gamma_{B}(P, K)=\Lambda_{B}(P, K)-\frac{1}{2} \int_{Q} \Lambda_{B}(P, Q) D_{B}^{2}(Q) \Gamma_{B}(Q, K)
$$

where

$$
\Lambda_{B}(P, K)=4 \frac{\delta^{2} \Phi\left[\lambda_{B}, D_{B}\right]}{\delta D_{B}(P) \delta D_{B}(K)} .
$$

In terms of renormalized quantities, this is simply

$$
\Gamma(P, K)=\Lambda(P, K)-\frac{1}{2} \int_{Q} \Lambda(P, Q) D^{2}(Q) \Gamma(Q, K),
$$

with

$$
\Lambda(P, K)=Z^{2} \Lambda_{B}(P, K)=4 \frac{\delta^{2} \Phi\left[Z_{\lambda} \lambda, D\right]}{\delta D(P) \delta D(K)}
$$

Note that $\Lambda$ is not a finite quantity: as we shall see, it contains coupling constant counterterms which are needed to remove the divergences in the integral equation for $\Gamma$.

Related to the Bethe-Salpeter equation is the equation for $\partial \Pi / \partial m^{2}$. In terms of bare quantities this is Eq. (11.18). For the renormalized theory we have:

$$
m^{2} \frac{\partial \Pi(K)}{\partial m^{2}}=-\frac{m^{2}}{2} \int_{Q} \Lambda(K, Q) D^{2}(Q)\left(1+\frac{\partial \Pi(Q)}{\partial m^{2}}\right)+\delta m^{2}
$$

where we have used the fact that $\delta m^{2} / m^{2}$ can be chosen to be a constant independent of $m^{2}$. This equation will be used to determine the mass counterterm from the requirement that $\partial \Pi / \partial m^{2}$ be finite.

Let us now summarize the procedure that we shall follow in order to identify properly the subdivergences. We shall expand the self-energy in powers of the coupling constant, keeping at a given order only those diagrams which are generated by iterating the gap equation. Then we shall perform a standard analysis of the subdivergences and show 
that they can be eliminated order by order by appropriate local counterterms. We shall be helped in this analysis by the consideration of the Bethe-Salpeter equation which will lead to the determination of the coupling constant counterterm $\delta \lambda$, and the equation for $\partial \Pi / \partial m^{2}$ which will fix the mass counterterm $\delta m^{2}$. The last counterterm, $\delta Z$, associated with field normalization, will be obtained independently by considering an equation for the asymptotic part of the propagator. Once the counterterms are known, one can verify that the gap equation is finite, and we shall present a well defined procedure to obtain such an equation. One can also verify that the temperature dependent infinities go away: these appear typically as products of finite temperature contributions, which are finite, by zero temperature subdiagrams, which are infinite, but are renormalized by zero temperature counterterms. In summary, once the subdivergences have been properly eliminated, all apparent temperature dependent divergences are also eliminated.

\section{THE TWO-LOOP APPROXIMATION}

In this section, we discuss the approximation obtained by keeping in $\Phi$ only the twoloop skeleton. The presentation is more detailed than needed for the solution of the problem, which is well known [13, 14]. However, many features of the renormalization of a self-consistent approximation already appear in this simple example and can be made explicit with analytical calculations. This two-loop example constitutes therefore an excellent preparation for the generalizations to be done in the next sections.

The contribution of the two-loop skeleton is

$$
\Phi=\frac{\lambda_{B}}{8}\left(\int_{P} D_{B}(P)\right)^{2}=\frac{\lambda+\delta \lambda}{8}\left(\int_{P} D(P)\right)^{2} .
$$

The self-energy, obtained from Eq. (II.31), reads then:

$$
\Pi=\frac{\lambda+\delta \lambda}{2} \int_{P} D(P)+\delta m^{2}
$$

where $D^{-1}(P)=P^{2}+m^{2}+\Pi$, and $\Pi$ is in this case a constant (so that $\delta Z=0$ and $\left.D_{B}=D\right)$. Finally the kernel of the Bethe-Salpeter equation is

$$
\Lambda=\lambda_{B}=\lambda+\delta \lambda
$$


and the Bethe-Salpeter equation itself reads:

$$
\Gamma=\Lambda-\frac{\Lambda}{2} \Gamma \int_{P} D^{2}(P)
$$

with $\Gamma$ a constant. This equation can be conveniently rewritten in the form:

$$
\frac{1}{\Gamma}=\frac{1}{\Lambda}+\frac{1}{2} \int_{P} D^{2}(P)
$$

The integrals above are ultraviolet divergent. We define them through dimensional regularisation, with $d=4-2 \epsilon$ and $\mu$ the renormalization scale. The dimension of the 4-point functions $\Lambda$ and $\Gamma$ becomes then that of the bare coupling constant $\lambda_{B}$ in $d$ dimensions, i.e., $\mu^{2 \epsilon}$. It is convenient to rescale these functions so as to keep them dimensionless:

$$
\Lambda \longrightarrow \mu^{2 \epsilon} \Lambda, \quad \Gamma \longrightarrow \mu^{2 \epsilon} \Gamma, \quad \lambda_{B} \longrightarrow \mu^{2 \epsilon}(\lambda+\delta \lambda)
$$

The Bethe-Salpeter equation reads then as in Eq. (III.4), with a factor $\mu^{2 \epsilon}$ multiplying the integral. As for the gap equation it becomes

$$
\Pi=\frac{\Lambda}{2} \mu^{2 \epsilon} \int_{P} D(P)+\delta m^{2}
$$

and $\Pi$ has the same dimension as $m^{2}$.

The foregoing analysis will involve Feynman diagrams calculated with the free propagator: $D_{0}(P)=\left(P^{2}+m^{2}\right)^{-1}$. The needed integrals are (with $\left.\bar{\mu}^{2}=4 \pi \mathrm{e}^{-\gamma_{E}} \mu^{2}\right)$ :

$$
\mathcal{B}_{0}\left(m^{2}\right) \equiv \mu^{2 \epsilon} \int_{P} D_{0}(P)=-\frac{m^{2}}{16 \pi^{2}}\left\{\frac{1}{\epsilon}-\ln \frac{m^{2}}{\bar{\mu}^{2}}+1\right\} \equiv \frac{a_{0}}{\epsilon}+b_{0},
$$

and

$$
\mathcal{B}_{1}\left(m^{2}\right) \equiv \mu^{2 \epsilon} \int_{P} D_{0}^{2}(P)=\frac{1}{16 \pi^{2}}\left\{\frac{1}{\epsilon}-\ln \frac{m^{2}}{\bar{\mu}^{2}}\right\}=-\frac{\partial \mathcal{B}_{0}}{\partial m^{2}} \equiv \frac{a_{1}}{\epsilon}+b_{1} .
$$

Note that $\mathcal{B}_{1}$ is dimensionless and that its divergent piece $a_{1} / \epsilon$ is independent of $m$ $\left(a_{1}=1 / 16 \pi^{2}\right)$.

At finite temperature, these two integrals receive additional finite contributions, respectively $b_{0}^{T}$ and $b_{1}^{T}$, with

$$
b_{0}^{T}=\int \frac{\mathrm{d}^{3} p}{(2 \pi)^{3}} \frac{n\left(\epsilon_{p}\right)}{\epsilon_{p}}
$$


and $b_{1}^{T}=-\partial b_{0}^{T} / \partial m^{2}$.

Before we proceed, let us comment on a typical difficulty that we are faced with. Since the divergence in Eq. (III.2) is a local one, one could, naively, attempt to absorb it in the mass counterterm $\delta m^{2}$. However, if one does this, one obtains a counterterm whose divergent part depends on the self-energy $\Pi$. This is not desirable since, at finite temperature, $\Pi$ depends on the temperature, and that would introduce temperature dependent counterterms in the calculation. Besides, one expects on general grounds to be a ble to put the mass counterterm in the form $\delta m^{2}=m^{2} C(\lambda, \epsilon)$, with $C$ independent of $m$ [15]. Clearly, the $\Pi$-dependent mass counterterm just defined would not have this property, as easily seen by taking a derivative with respect to $m^{2}$. In fact the infinities in Eq. (III.2) result from subdivergences which are best exhibited by iterating the gap equation. And to properly identify $\delta \lambda$ and $\delta m^{2}$, one needs to carefully disentangle these various subdivergences.

We are therfore led to analyze the formal solutions of the gap equation (III.7), and of the Bethe-Salpeter equation (III.4), that are obtained by the successive iterations of these equations. Such iterations construct the solutions as powers series in the coupling constant $\lambda$. These series coincide with those one would obtain by applying the rules of ordinary perturbation theory, and restricting the Feynman diagrams to belong to a certain topology. The solutions after $n$ iterations, to be denoted respectively by $\Pi^{(n)}$ and $\Gamma^{(n)}$, are of order $\lambda^{n+1}$, with each iteration bringing in a new power of $\lambda$, and associated new divergences. The renormalization consists in showing that, given the mass and coupling constant counterterms determined at the previous iterations, of order $\lambda^{n}$ at most, the new divergences can be absorbed in a new contribution to these counterterms, of order $\lambda^{n+1}$. Note that whether this can be done or not is not guaranteed a priori by the standard proof of perturbative renormalizability, since at any order $\lambda^{n}$ only a subset of terms contributing at this order is included. 


\section{A. Iterative solution of the Bethe-Salpeter equation}

We consider first the Bethe-Salpeter equation (III.4) in which we replace the propagator $D$ by the free propagator $D_{0}$. We call $\Gamma_{0}$ the corresponding 4-point function, that is:

$$
\Gamma_{0}=\Lambda-\frac{\Lambda}{2} \Gamma_{0} \mu^{2 \epsilon} \int_{P} D_{0}^{2}(P)=\Lambda-\frac{\Lambda}{2} \Gamma_{0} \mathcal{B}_{1}\left(m^{2}\right) .
$$

As we have just mentioned, we shall construct the counterterms by building the solution of Eq. (III.11) as a formal series in powers of the coupling constant $\lambda$. This is obtained by iteration, keeping at each iteration $k$ only terms which contribute to $\Gamma_{0}$ up to order

$\lambda^{k+1}$. Thus, $\Gamma_{0}^{(n)}$, the value of $\Gamma_{0}$ obtained after $n$ iterations, is of order $\lambda^{n+1}$, and so is the counterterm $\delta \lambda_{n}$ that needs to be adjusted at iteration $n$. Clearly, we can write

$$
[\Lambda]_{[n+1]}=\lambda+\sum_{k=1}^{n} \delta \lambda_{k} .
$$

where $\delta \lambda_{k}$ is the counterterm of order $\lambda^{k+1}$. The notation $[\cdots]_{[n]}$, to be used throughout, indicates that only the terms up to order $\lambda^{n}$ are to be kept within the brakets.

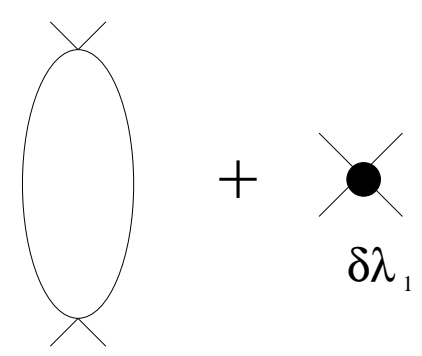

FIG. 7: The counterterm $\delta \lambda_{1}$ eliminates the divergence of the one-loop contribution to the 4-point function $\Gamma_{0}^{(1)}$.

In leading order, the solution $\Gamma_{0}^{(0)}$ is simply the renormalized coupling constant

$$
\Gamma_{0}^{(0)}=\lambda
$$

The first iteration gives

$$
\Gamma_{0}^{(1)}=\lambda+\delta \lambda_{1}-\lambda^{2} \frac{\mathcal{B}_{1}}{2}=\lambda+\delta \lambda_{1}-\frac{\lambda^{2}}{2}\left(\frac{a_{1}}{\epsilon}+b_{1}\right) .
$$


As anticipated, there is a divergent contribution given by the one-loop diagram in Fig. 7. This divergence is absorbed in the counterterm $\delta \lambda_{1}$. Using minimal subtraction, one gets:

$$
\delta \lambda_{1}=\frac{\lambda^{2} a_{1}}{2 \epsilon} .
$$

Note that we have ignored in (III.14) the terms $\sim \lambda \delta \lambda_{1}$ and $\sim \delta \lambda_{1}^{2}$ which are of order higher than $\lambda^{2}$. Note also that $\Gamma_{0}^{(1)}$ is only a part of the 4-point function that would be obtained in perturbation theory at order $\lambda^{2}$. This is so because, as we have already mentioned, the Bethe-Salpeter equation performs a resummation in only one out of three possible channels. The contributions of other channels are recovered only when included in the kernel $\Lambda$ (see next section).

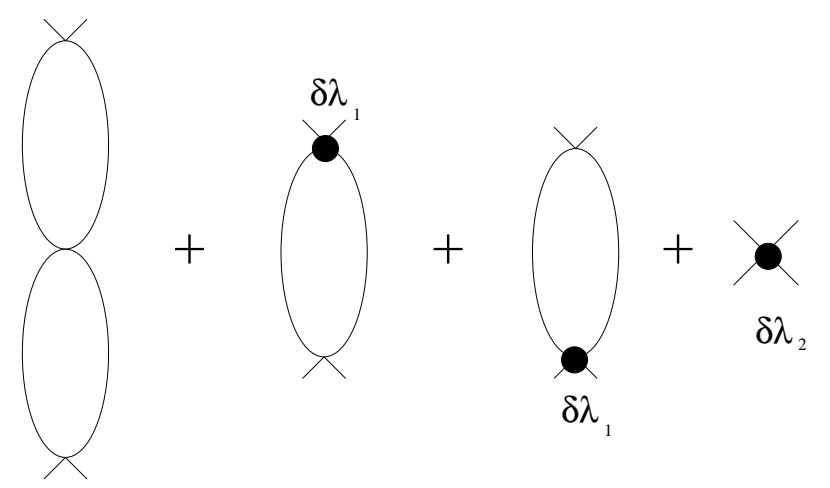

FIG. 8: The counterterm $\delta \lambda_{2}$ eliminates the global divergence which remains in the two-loop contribution to $\Gamma_{0}^{(2)}$ (diagram on left) after the subdivergences have been removed by the two middle diagrams involving $\delta \lambda_{1}$.

Doing the second iteration and keeping only terms up to order $\lambda^{3}$, one obtains

$$
\begin{aligned}
\Gamma_{0}^{(2)} & =\left[\Lambda-\Lambda \frac{\mathcal{B}_{1}}{2} \Gamma_{0}^{(1)}\right]_{[3]} \\
& =\lambda+\delta \lambda_{1}+\delta \lambda_{2}-\lambda\left(\lambda+2 \delta \lambda_{1}\right) \frac{\mathcal{B}_{1}}{2}+\lambda^{3} \frac{\mathcal{B}_{1}^{2}}{4},
\end{aligned}
$$

where the notation $[\cdots]_{[n]}$ is that of Eq. (III.12). Note that although there is a single counterterm to adjust, namely $\delta \lambda_{2}$, one needs to keep combinations of terms which where ignored at the previous iteration, such as $\lambda \delta \lambda_{1}$ : such terms are needed to remove the 
subdivergences that are now present, as can be seen in the diagrams displayed in Fig. 8. Observe that in the cancellation of subdivergences, the term involving the product of $\delta \lambda_{1}$ and the finite part of $\mathcal{B}_{1}$ also cancels. The global divergence that remains, and that is absorbed in $\delta \lambda_{2}$, depends therefore only on $a_{1}$. A simple calculation gives

$$
\delta \lambda_{2}=\lambda\left(\frac{\lambda a_{1}}{2 \epsilon}\right)^{2}
$$

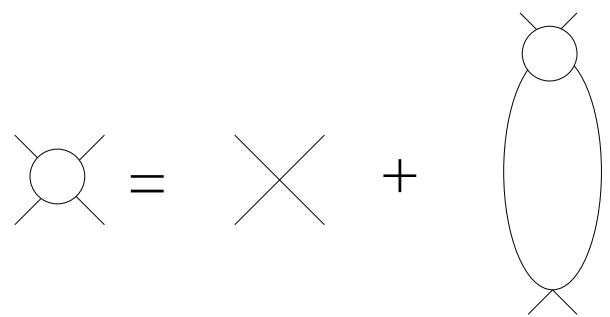

FIG. 9: Diagrammatic interpretation of the Bethe-Salpeter equation.

Quite generally, at iteration $n$ one gets

$$
\Gamma_{0}^{(n)}=\left[\Lambda-\Lambda \Gamma_{0}^{(n-1)} \frac{\mathcal{B}_{1}}{2}\right]_{[n+1]} .
$$

The term $\Gamma_{0}^{(n-1)}$ is assumed to be finite. It contains all the counterterms which eliminate the subdivergences that do not involve the lowest vertex in Fig. 9. The factor $\Lambda$ multiplying it brings the counterterms needed to remove the subdivergences involving the lower vertex. After all the subdivergences have been removed by the counterterms determined in the $(n-1)$ previous iterations, there remains a global divergence which is cancelled by the adjustment of the contribution $\delta \lambda_{n}$ to $\Lambda$ (which affects only the first $\Lambda$ in the r.h.s. of Eq. (1II.18)). An illustration at order $\lambda^{3}$ is provided in Fig. 10,

One can rewrite Eq. (III.18) as follows:

$$
\Gamma_{0}^{(n)}=\left[\frac{\Lambda}{1+\Lambda \frac{\mathcal{B}_{1}}{2}}\right]_{[n+1]}=\left[\Gamma_{0}\right]_{[n+1]} .
$$

The last equality emphasizes the fact that $\Gamma_{0}^{(n)}$ is nothing but the perturbative solution of order $\lambda^{n+1}$, restricted to the particular resummation of the chain of bubbles in the 


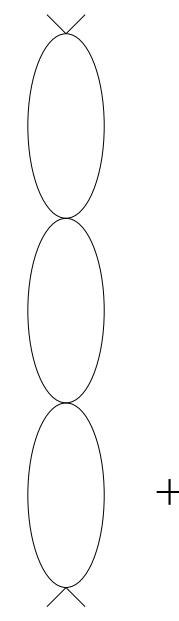

(a)

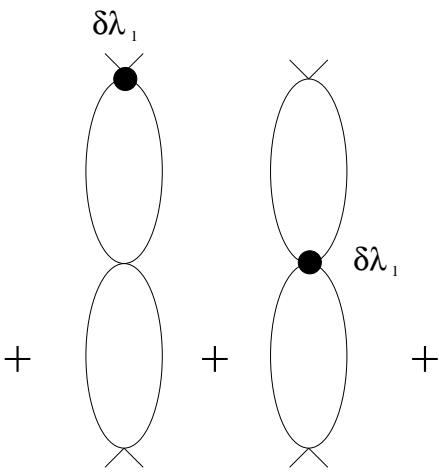

(b) (c)

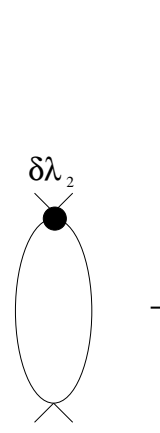

(d)

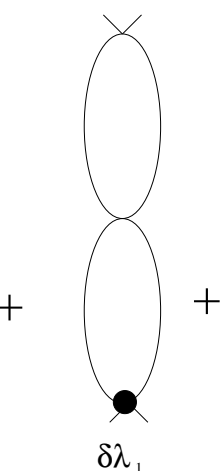

(e)

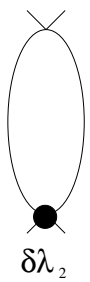

(f)

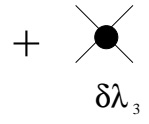

$\delta \lambda_{3}$

FIG. 10: Contributions to $\Gamma_{0}^{(2)}$ at order $\lambda^{3}$, illustrating the elimination of the various subdivergences of the diagram (a) by counterterms determined at the first two iterations (i.e., $\delta \lambda_{1}$ and $\left.\delta \lambda_{2}\right)$; these leave a global divergence absorbed in $\delta \lambda_{3}$. Diagrams (b,c,d) represent counterterms for subdivergences which do not involve the lower vertex of (a); diagrams (e) and (f) eliminate the subdivergences involving the lower vertex of (a).

$t$-channel. In order to determine the general form of the counterterms, it is in fact more convenient to invert this relation:

$$
[\Lambda]_{[n+1]}=\left[\frac{\Gamma_{0}}{1-\Gamma_{0} \frac{\mathcal{B}_{1}}{2}}\right]_{[n+1]},
$$

and exploit the fact that $\Gamma_{0}$ is finite. Then, it is not hard to get

$$
\lambda=\frac{\Gamma_{0}}{1-\frac{b_{1}}{2} \Gamma_{0}}, \quad \delta \lambda_{n}=\lambda\left(\frac{\lambda a_{1}}{2 \epsilon}\right)^{n},
$$

where $a_{1}$ and $b_{1}$ are defined in Eq. (III.9).

At this point we note that the counterterms depend only on $a_{1}$, that is, they are independent of the mass. It follows that the Bethe-Salpeter equation (III.11) written with the full propagator $D$ instead of the free propagator $D_{0}$, can be made finite with the same counterterms as those determined for $\Gamma_{0}$. This may also be seen by observing that 
any insertion of a self-energy in the propagators of the Bethe-Salpeter equation alters only the finite part of the integral in Eq. (III.4).

Let then $\Gamma$ be the solution obtained with the full propagator $D$. From the first of Eqs. (III.21), and a similar equation for $\Gamma$ (obtained by replacing simply $m^{2}$ by $m^{2}+\Pi$ ), one easily gets

$$
\frac{1}{\lambda}=\frac{1}{\Gamma_{0}}-\frac{1}{2} b_{1}\left(m^{2}\right)=\frac{1}{\Gamma}-\frac{1}{2} b_{1}\left(m^{2}+\Pi\right) .
$$

Thus, $\Gamma$ and $\Gamma_{0}$ differ indeed only by a finite part:

$$
\frac{1}{\Gamma}-\frac{1}{\Gamma_{0}}=\frac{1}{32 \pi^{2}} \ln \frac{m^{2}}{m^{2}+\Pi},
$$

where we have used the explicit form of $b_{1}\left(m^{2}\right)$ given in Eq. ([II.9).

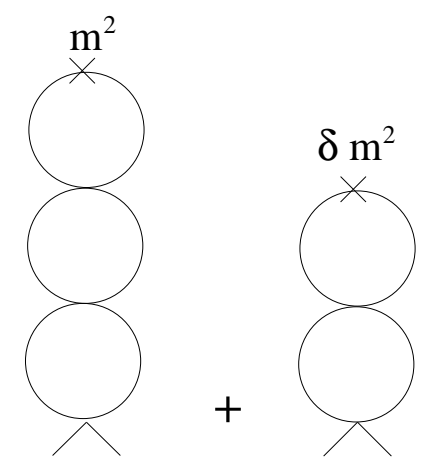

FIG. 11: Diagrammatic representation of the two terms in the right hand side of Eq. (III.24).

\section{B. The equation for the mass counterterm}

We now turn to the equation satisfied by $\partial \Pi / \partial m^{2}$, whose structure, as discussed in Sect. II, is very close to that of the Bethe-Salpeter equation. This will allow us to determine the counterterm $\delta m^{2}$, which we assume of the form $\delta m^{2}=m^{2} C(\lambda, \epsilon)$. From Eq. (II.19) (or directly from Eq. (III.2) ), one easily gets:

$$
m^{2} \frac{\partial \Pi}{\partial m^{2}}=-\left(1+\frac{\Lambda}{2} \mathcal{B}_{1}\right)^{-1} \frac{\Lambda}{2} \mathcal{B}_{1} m^{2}+\left(1+\frac{\Lambda}{2} \mathcal{B}_{1}\right)^{-1} \delta m^{2},
$$


where $\mathcal{B}_{1}$ stands here for $\mathcal{B}_{1}\left(m^{2}+\Pi\right)$. This equation has a simple diagrammatic interpretation: each term represents a tower of bubbles, with $m^{2}$ or $\delta m^{2}$ inserted in the upper most bubble (see Fig. 11). The corresponding diagrams are easily generated by expanding the right hand side of this equation in powers of $\lambda$. A simple analysis then reveals that the subdivergences associated with coupling constant counterterms cancel out; that is, the potential subdivergences are removed by the same counterterms as introduced in solving the Bethe-Salpeter equation. Such subdivergences in the towers of bubbles of Fig. [1] are those which do not involve the upper loop with $m^{2}$ or $\delta m^{2}$ inserted. The subdivergences involving the upper loops are remove by adjusting the mass counterterm $\delta m^{2}$ at each iteration. The elimination of coupling constant subdivergences can be made explicit by using the Bethe-Salpeter equation (III.19) to write:

$$
m^{2} \frac{\partial \Pi}{\partial m^{2}}=-\frac{\Gamma}{2} \mathcal{B}_{1} m^{2}+\left(1-\frac{\Gamma}{2} \mathcal{B}_{1}\right) \delta m^{2},
$$

where we have used the identity

$$
\left(1+\frac{\Lambda}{2} \mathcal{B}_{1}\right)^{-1}\left(1-\frac{\Gamma}{2} \mathcal{B}_{1}\right)=1 .
$$

The mass counterterms can then be easily determined by expanding the r.h.s. of Eq. (III.25) in powers of $\lambda$, identifying at each order the divergent part of $\delta m^{2}$. In leading order we get

$$
\delta m_{0}^{2}=m^{2} \frac{\lambda a_{1}}{2 \epsilon}
$$

At next order, we have:

$$
m^{2} \frac{\partial \Pi^{(1)}}{\partial m^{2}}=-m^{2} \frac{\Gamma^{(1)}}{2} \mathcal{B}_{1}+\delta m_{0}^{2}\left(1-\frac{\lambda}{2} \mathcal{B}_{1}\right)+\delta m_{1}^{2},
$$

where $\Pi^{(1)}$ and $\Gamma^{(1)}$ are the values of $\Pi$ and $\Gamma$ obtained at the first iteration of, respectively, the gap equation and the Bethe-Salpeter equation. Keeping only the divergent parts of order $\lambda^{2}$ one gets

$$
\begin{aligned}
\frac{\delta m_{1}^{2}}{m^{2}} & =\left[\left(\delta \lambda_{1}-\frac{\lambda^{2}}{2} \mathcal{B}_{1}\right) \frac{\mathcal{B}_{1}}{2}\right]_{d i v}+\lambda \frac{\delta m_{0}^{2}}{m^{2}} \frac{\mathcal{B}_{1}}{2} \\
& =\left(-\frac{\lambda^{2}}{2} b_{1}\right) \frac{a_{1}}{2 \epsilon}+\frac{\delta m_{0}^{2}}{m^{2}}\left(\frac{\lambda a_{1}}{2 \epsilon}+\frac{\lambda b_{1}}{2}\right),
\end{aligned}
$$


where we have used Eq. (III.14) for $\Gamma^{(1)}$. Observe the cancellation of the term involving the leading order mass counterterm $\delta m_{0}^{2}$ multiplied by the finite part $b_{1}$, leaving the following formula for $\delta m_{1}^{2}$ :

$$
\begin{aligned}
\frac{\delta m_{1}^{2}}{m^{2}} & =\frac{\delta m_{0}^{2}}{m^{2}}\left(\frac{\lambda a_{1}}{2 \epsilon}\right) \\
& =\frac{\delta \lambda_{1}}{2} \frac{a_{1}}{\epsilon}
\end{aligned}
$$

In the second line we have written the formula which emerges naturally if one cancels the subdivergences in a different order (making use of the explicit expression (III.27) of $\left.\delta m_{0}\right)$. This writing generalizes to high orders. This can be seen by rewriting Eq. (III.25) as follows:

$$
m^{2} \frac{\partial \Pi}{\partial m^{2}}=-m^{2} \frac{\Gamma}{2} \mathcal{B}_{1}+\frac{\Gamma}{\Lambda} \delta m^{2}
$$

Clearly, the divergence in the right hand side is cancelled if we choose

$$
\frac{\delta m^{2}}{m^{2}}=\frac{\Lambda}{2} \frac{a_{1}}{\epsilon}
$$

We have obtained earlier $\Lambda$ as a power series in the coupling constant (see Eqs. (III.12) and (III.21) $)$. Similarly we can write

$$
\left[\delta m^{2}\right]_{[n+1]}=\sum_{k=0}^{n} \delta m_{k}^{2}
$$

with

$$
\delta m_{k}^{2}=m^{2}\left(\frac{\lambda a_{1}}{2 \epsilon}\right)^{k+1} .
$$

As was the case for the Bethe-Salpeter equation, it does not matter for the determination of the divergent part of the mass counterterm whether the free propagator $D_{0}$, or the full propagator $D$ is used in Eq. (III.31): this is a priori clear from the diagrammatic structure in Fig. 111 and is obvious in Eq. (111.32). But Eqs. (III.24) or (III.31) for $\partial \Pi / \partial m^{2}$, including the finite parts, hold only if the full propagator is used (as is clear from their derivation). 


\section{Iterative solution of the gap equation}

We turn now to the gap equation (III.7), and construct iteratively its solution as a formal series in powers of the coupling constant $\lambda$. At a given order in $\lambda$, $\Pi$ contains two types of subdivergences: those associated with the renormalization of the coupling constant, and those associated with the renormalization of the mass.

We shall verify that the counterterms $\delta \lambda_{n}$ determined in solving the Bethe-Salpeter equation eliminate the subdivergence of the first kind, while those of the second kind are eliminated by the mass counterterm $\delta m_{n}^{2}$ obtained at the end of the previous subsection.

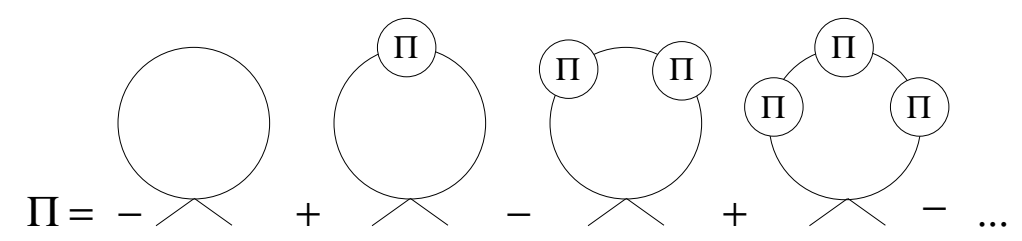

FIG. 12: Diagrammatic interpretation of the expansion (III.35)

We start by expanding the r.h.s. of the gap equation in powers of $\Pi$ :

$$
\begin{aligned}
\Pi & =\frac{\Lambda}{2} \int_{P} \frac{1}{P^{2}+m^{2}+\Pi}+\delta m^{2} \\
& =\frac{\Lambda}{2} \sum_{k=0}(-1)^{k} \mathcal{B}_{k} \Pi^{k}+\delta m^{2} .
\end{aligned}
$$

The coefficients $\mathcal{B}_{k}$ are given by

$$
\mathcal{B}_{k}=\mu^{2 \epsilon} \int_{P} \frac{1}{\left(P^{2}+m^{2}\right)^{k+1}}=\frac{(-)^{k}}{k !} \frac{\partial^{k} B_{0}}{\partial\left(m^{2}\right)^{k}} .
$$

The expansion (III.35) is represented diagrammatically in Fig 12 Note that only $\mathcal{B}_{0}$ and $\mathcal{B}_{1}$ are ultraviolet divergent and, as we have seen in the previous subsection, in minimal subtraction the counterterms $\delta \lambda$ and $\delta m^{2}$ depend only on their divergent parts, respectively $a_{0}$ and $a_{1}$. Each iteration brings in one power of $\lambda$ so that $\Pi^{(n)}$, the value of $\Pi$ at iteration $n$, is of order $\lambda^{n+1}$. This means that at iteration $n \geq 1$ one can stop the expansion (III.35) at $k=n$. 
The lowest order contribution, $\Pi^{(0)}$, is that of the 1-loop diagram in Fig 13

$$
\Pi^{(0)}=\frac{\lambda}{2} \mathcal{B}_{0}+\delta m_{0}^{2}=\frac{\lambda}{2}\left(\frac{a_{0}}{2}+b_{0}\right)+\delta m_{0}^{2}
$$

There is no subdivergence in this diagram and the global divergence is absorbed in the mass counterterm $\delta m_{0}^{2}$ given in Eq. (III.27) (recall that $a_{0}=-m^{2} a_{1}$ ).

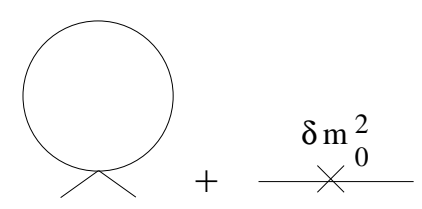

FIG. 13: The 1-loop contribution to $\Pi^{(0)}$, and the corresponding mass counterterm.

The first iteration of the gap equation, in which we keep only the terms up to order $\lambda^{2}$, leads to

$$
\begin{aligned}
\Pi^{(1)} & =\left[-\frac{\Lambda}{2} \mathcal{B}_{1} \Pi^{(0)}\right]_{[2]}+\frac{1}{2}[\Lambda]_{[2]} \mathcal{B}_{0}+\left[\delta m^{2}\right]_{[2]} \\
& =\frac{\lambda}{2} \mathcal{B}_{0}+\delta m_{0}^{2}+\frac{\mathcal{B}_{0}}{2}\left(\delta \lambda_{1}-\frac{\lambda^{2}}{2} \mathcal{B}_{1}\right)-\delta m_{0}^{2} \frac{\lambda}{2} \mathcal{B}_{1}+\delta m_{1}^{2}
\end{aligned}
$$

In the second line we have grouped the terms so as to display the cancellation of divergences: one recognizes indeed in the last four terms, of order $\lambda^{2}$, the structure already met in Eq. (ㅍ.28) for $\partial \Pi / \partial m^{2}$ (recall that the divergent part of $\mathcal{B}_{0}$ is the same as that of $\left.m^{2} \mathcal{B}_{1}\right)$. This is illustrated in Fig. 14,

A further iteration will allow us to uncover the generic features of the iteration procedure. Keeping only terms up to order $\lambda^{3}$ on obtains:

$$
\Pi^{(2)}=\frac{\lambda}{2} \mathcal{B}_{2}\left(\Pi^{(0)}\right)^{2}-\frac{\lambda}{2} \mathcal{B}_{1} \Pi^{(1)}-\frac{\delta \lambda_{1}}{2} \mathcal{B}_{1} \Pi^{(0)}+\frac{1}{2}[\Lambda]_{[3]} \mathcal{B}_{0}+\left[\delta m^{2}\right]_{[3]},
$$

This iteration adds to $\Pi$ a 3-loop contribution, whose corresponding diagrams are displayed in Fig 15, The first term in Eq. (III.39), quadratic in $\Pi$, is finite; all the divergences are localized in the terms linear in $\Pi$ and to isolate them we need to express $\Pi^{(0)}$ and $\Pi^{(1)}$ in terms of elementary integrals. Again, as was the case in the first iteration, we shall find that the counterterms determined at the previous two iterations eliminate all the 


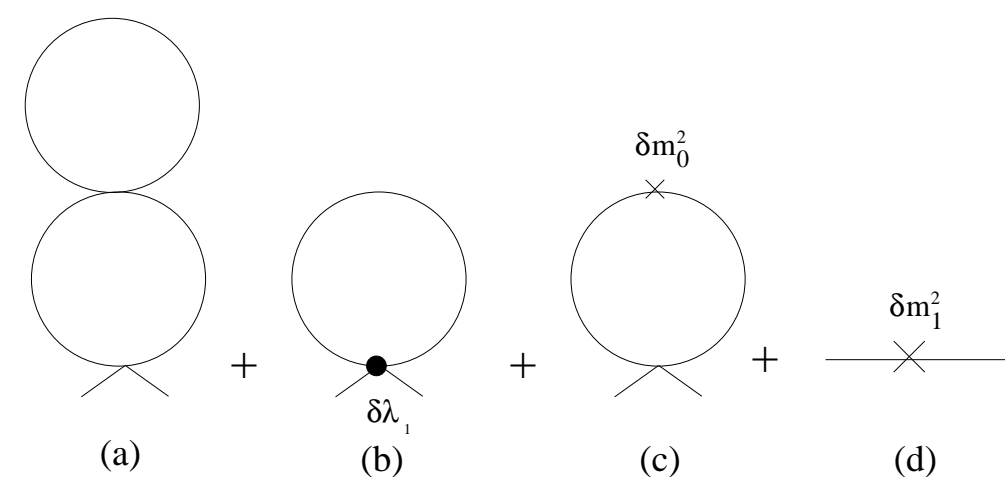

FIG. 14: The 2-loop contribution to $\Pi^{(1)}$, exhibiting two subdivergences. That associated with coupling constant renormalization (the lower loop) is eliminated by diagram (b) involving $\delta \lambda_{1}$; that associated with mass renormalization (the upper loop in (a)) is eliminated by diagram (c) involving $\delta m_{0}^{2}$. The remaining global divergence is absorbed in the mass counterterm $\delta m_{1}^{2}$.

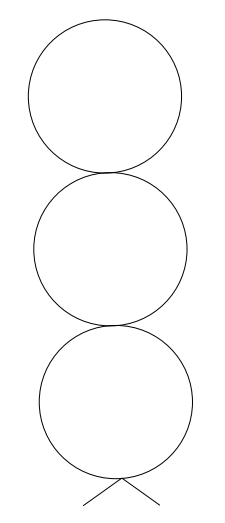

(a)

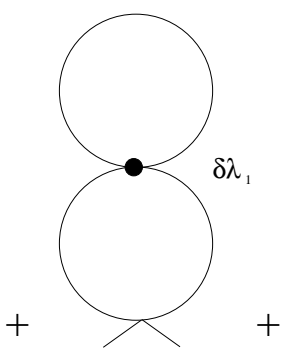

(b)

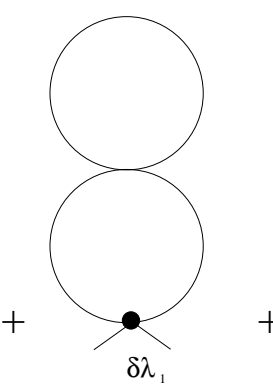

(c)

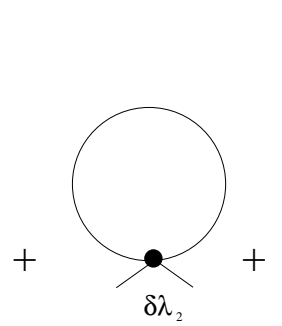

(d)

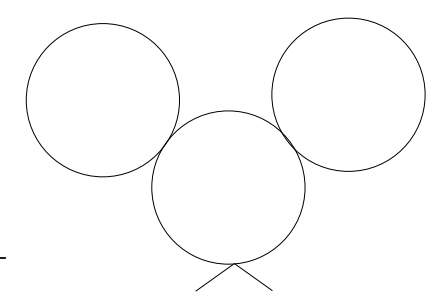

(e)

FIG. 15: The 3-loop contribution to $\Pi^{(2)}$, with its coupling constant counterterms (mass counterterms are not drawn).

subdivergences but one, namely that associated with diagram (a). This subdivergence is recognized as the global divergence of the order $\lambda^{3}$ contribution to the 4-point function and it is absorbed in $\delta \lambda_{2}$. The remaining divergence is a global one and is eliminated by new mass counterterm $\delta m_{2}^{2}$.

It is useful to observe that the new divergences which enter at each new iteration $n$, 
and which require the new counterterms $\delta \lambda_{n}$ and $\delta m_{n}^{2}$, are entirely contained in the tower of bubbles such as diagram (a) in Fig. 15. The other structures which develop as we proceed through the iterations, such as diagram (e) in Fig. 15, are all made finite by previously determined counterterms (for diagram (e) this is due to the mass counterterm that is not drawn here). Then, it is not hard to see that all the divergences in such a tower cancel in exactly the same way as they do in the equation for $\partial \Pi / \partial m^{2}$. To make this explicit, note that we can rewrite Eq. (III.39) as follows

$$
\Pi^{(2)}=\frac{\lambda}{2} \mathcal{B}_{2}\left(\Pi^{(0)}\right)^{2}+\left[\left(1+\frac{\Lambda}{2} \mathcal{B}_{1}\right)^{-1} \frac{\Lambda}{2}\right]_{[3]} \mathcal{B}_{0}+\left[\left(1+\frac{\Lambda}{2} \mathcal{B}_{1}\right)^{-1} \delta m^{2}\right]_{[3]}
$$

The last two terms represent the contribution to $\Pi^{(2)}$ obtained by iterating only the term linear in $\Pi$, thus generating the tower of bubbles such as that in Fig. 15(a), together with the counterterms which make it finite. That the contribution of these last two terms of Eq. (III.40) is finite is made clear by comparing them to the right hand side of (III.24), observing that factor in front of $\mathcal{B}_{0}$ in Eq. (III.40) is $\Gamma / 2$ (see also Eq. (III.25) ), and that the divergent parts of $\mathcal{B}_{0}$ and $-m^{2} \mathcal{B}_{1}$ are the same.

One can summarize the iterative procedure by the following formula giving the solution $\Pi^{(n)}$ at iteration $n$ in terms of the (finite) solution $\Pi^{(n-1)}$ obtained at iteration $(n-1)$ :

$$
\Pi^{(n)}=\left[\frac{\Lambda}{2} \sum_{k=1}^{n}(-1)^{k} \mathcal{B}_{k}\left(\Pi^{(n-1)}\right)^{k}\right]_{[n+1]}+[\Lambda]_{[n+1]} \frac{\mathcal{B}_{0}}{2}+\left[\delta m^{2}\right]_{[n+1]},
$$

where we have separated the terms which contain $\Pi^{(n-1)}$ from those which contain only the new counterterms that need to be introduced at each iteration, and which are independent of $\Pi$. It will prove convenient to introduce a special notation for these two contributions. We set:

$$
\begin{gathered}
\tilde{\Pi}_{0}^{(n)}=\left[\frac{\Lambda}{2} \sum_{k=1}^{n}(-1)^{k} \mathcal{B}_{k}\left(\Pi^{(n-1)}\right)^{k}\right]_{[n+1]} \\
\tilde{\Pi}_{2}^{(n)}=[\Lambda]_{[n+1]} \frac{\mathcal{B}_{0}}{2}+\left[\delta m^{2}\right]_{[n+1]}
\end{gathered}
$$

so that $\Pi^{(n)}=\tilde{\Pi}_{0}^{(n)}+\tilde{\Pi}_{2}^{(n)}$. 
From the previous analysis, we expect the new subdivergences to occur in the term with $k=1$. However, we have to make sure that subdivergences introduced by the factor $\Lambda$ in front of otherwise finite integrals do cancel. In order to do so, we use Eq. (III.18) to express $\Lambda$ in terms of $\Gamma_{0}$ within Eq. (III.42), and obtain:

$$
\begin{aligned}
\tilde{\Pi}_{0}^{(n)} & =\left[\frac{\Gamma_{0}}{2} \sum_{k=1}^{n}(-1)^{k} \mathcal{B}_{k}\left(\Pi^{(n-1)}\right)^{k}+\frac{\Gamma_{0}}{2} \mathcal{B}_{1} \tilde{\Pi}_{0}^{(n-1)}\right]_{[n+1]} \\
& =\left[\frac{\Gamma_{0}}{2} \sum_{k=2}^{n}(-1)^{k} \mathcal{B}_{k}\left(\Pi^{(n-1)}\right)^{k}+\frac{\Gamma_{0}}{2} \mathcal{B}_{1}\left(\tilde{\Pi}_{0}^{(n-1)}-\Pi^{(n-1)}\right)\right]_{[n+1]} .
\end{aligned}
$$

The first term is now finite. The divergences have been isolated in the last two terms where we can identify $\tilde{\Pi}_{0}^{(n-1)}-\Pi^{(n-1)}=-\tilde{\Pi}_{2}^{(n-1)}$. One then gets for $\Pi^{(n)}=\tilde{\Pi}_{0}^{(n)}+\tilde{\Pi}_{2}^{(n)}$ :

$$
\Pi^{(n)}=\left[\frac{\Gamma_{0}}{2} \sum_{k=2}^{n}(-1)^{k} \mathcal{B}_{k}\left(\Pi^{(n-1)}\right)^{k}\right]_{[n+1]}-\left[\frac{\Gamma_{0}}{2} \mathcal{B}_{1} \tilde{\Pi}_{2}^{(n-1)}\right]_{[n+1]}+\tilde{\Pi}_{2}^{(n)} .
$$

Using the definition (III.43) of $\tilde{\Pi}_{2}$, one can combine the last two terms as follows

$$
\left[\Lambda-\frac{\Gamma_{0}}{2} \mathcal{B}_{1} \Lambda\right]_{[n+1]} \frac{\mathcal{B}_{0}}{2}+\left[\left(1-\frac{\Gamma_{0}}{2} \mathcal{B}_{1}\right) \delta m^{2}\right]_{[n+1]} .
$$

But the divergent terms in this expression are those of the right hand side of Eq. (III.25) (see the discussion after Eq. (III.40) ). The two terms in Eq. (III.46) therefore add up to a finite quantity, $\left[\Gamma_{0}\right]_{[n+1]} b_{0} / 2$. We are then left with a finite equation for determining the finite part of $\Pi^{(n)}$ :

$$
\Pi^{(n)}=\left[\frac{\Gamma_{0}}{2} \sum_{k=2}^{n}(-1)^{k} \mathcal{B}_{k}\left(\Pi^{(n-1)}\right)^{k}\right]_{[n+1]}+\left[\Gamma_{0}\right]_{[n+1]} \frac{b_{0}}{2} .
$$

This completes the proof that $\Pi^{(n)}$ is made finite with the counterterms determined in the previous subsection.

\section{Algebraic solution and scheme dependence}

We now show that is is possible to obtain the previous solutions of both the BetheSalpeter equation and the gap equation by doing simple algebraic manipulations on the 
equations themselves, without going explicitly through the iterations. We shall call $M$ the physical mass, given by $M^{2}=m^{2}+\Pi$. Both $M$ and $\Gamma$ are physical quantities that are calculated as a function of the renormalized mass and coupling constant, $m$ and $\lambda$, using the self-consistent scheme.

First we note that the renormalized coupling constant $\lambda$ is related to the bare one $\lambda_{B}=\mu^{2 \epsilon} \Lambda$ by

$$
\frac{1}{\lambda}=\frac{1}{\Lambda}+\frac{a_{1}}{2 \epsilon}=\frac{1}{\Lambda}+\frac{1}{32 \pi^{2} \epsilon}
$$

This follows immediately form Eqs. (III.5) and (ㅍ․22). It can be also obtained directly from the Bethe Salpeter equation (III.11), observing that this equation is finite when expressed in terms of the renormalized coupling constant defined in Eq. (III.48). Since $\Lambda=\lambda+\delta \lambda$, Eq. (III.48) is equivalent to

$$
\delta \lambda=\frac{\lambda^{2} a_{1}}{2 \epsilon} \frac{1}{1-\frac{\lambda a_{1}}{2 \epsilon}}=\frac{\lambda^{2}}{32 \pi^{2} \epsilon} \frac{1}{1-\frac{\lambda}{32 \pi^{2} \epsilon}},
$$

which corresponds indeed to the series of counterterms that we have obtained via the diagrammatic analysis (cf. Eq. (III.21).

Using the fact that $\lambda_{B}=\mu^{2 \epsilon} \Lambda$ is independent of $\mu$, one easily obtains that $\lambda(\mu)$ is running with $\mu$ according to (we take the limit $\epsilon \rightarrow 0$ )

$$
\frac{d \lambda(\mu)}{d \ln \mu}=\frac{\lambda^{2}}{16 \pi^{2}} .
$$

Note that this relation is "exact" in the sense that it represents faithfully the relation between $\lambda_{B}$ and $\lambda$, to all orders in $\lambda$, within the class of diagrams which are taken into account in the present approximation. In this respect, it is instructive to verify how this relation holds as one iterates the Bethe-Salpeter equation in Sect. IIIA By demanding that the solution $\Gamma_{0}^{(n)}$ be independent of $\mu$ at iteration $n$ (at fixed $m^{2}$ ), the corrections to Eq. (III.50) are pushed to order $\lambda^{n+2}$; that is, potential corrections of order $\lambda^{n+1}$ cancel out, in very much the same way as subdivergences do. Now, while exact in the sense specified above, the $\beta$ function in Eq. (III.50) represents only one third of the perturbative one-loop $\beta$-function. This is so because, as already mentioned, 
the resummation performed by the one-loop gap equation involves the one-loop vertex correction in one channel only, namely the $t$-channel. The remaining two thirds of the one-loop $\beta$-function will be recovered after taking the 3-loop skeleton diagram into account (see next section).

Written as a function of the renormalized coupling constant, the renormalized 4-point function takes the form

$$
\Gamma=\frac{\lambda}{1-\frac{\lambda}{32 \pi^{2}} \ln \frac{M^{2}}{\bar{\mu}^{2}} .}
$$

Thus $\Gamma$ is nothing but the running coupling constant at the scale $\bar{\mu}=M$. One can verify that $\Gamma$ is independent of $\mu$ if $M$ is, which we shall verify soon. Note that $\lambda(\mu)$ diverges when $\ln \left(\bar{\mu}^{2} / M^{2}\right)=32 \pi^{2} / \Gamma$. The appearance of this "Landau pole" at large scales is a well known feature of $\phi^{4}$ field theory in four dimensions. Another manifestation of the same difficulty is visible on Eq. (III.49): when $\epsilon \rightarrow 0, \delta \lambda \rightarrow-\lambda$ or $\lambda_{B} \rightarrow 0$, reflecting the so-called "triviality" of the theory.

Knowing $\Gamma$, one could easily get a finite equation for $\partial \Pi / \partial m^{2}$. This is obtained from Eqs. (III.31) and (III.32):

$$
\frac{\partial \Pi}{\partial m^{2}}=-\frac{1}{2} \Gamma b_{1}\left(M^{2}\right) .
$$

However, it is not easy to exploit this equation to obtain a finite equation for $\Pi$ itself, by using methods that we could export to the general case. We shall therefore base our construction of a finite gap equation on the iterative solution obtained in the previous subsection.

There are two observations which will guide this construction. The first observation is that, in the iterative solution, the cancellation of ultraviolet divergences only takes place up to some order dictated by the number of iterations that have been completed: at iteration $n$ new divergences appear, of order $\lambda^{n+1}$, and some are ignored because they are of order higher than $\lambda^{n+1}$ (these high order contributions reenter the calculation as countertems needed to cancel subdivergences in the following iterations). Thus, in an explicit algebraic solution, one should expect the cancellation of divergences to occur 
only for the solution of the gap equation, which effectively sums up all the iterations. The second observation concerns the particular role of the term linear in $\Pi$ in the expansion of the right hand side of the gap equation in powers of $\Pi$ (see Eq. (III.35) ). As we have seen, the new divergences in the gap equation at each new iteration come from diagrams which are obtained by iterating just this term (e.g. the towers of bubbles in Fig. 15). Terms with several insertions of $\Pi$ on the same propagator are finite (assuming that such insertions have been made finite in previous iterations). With these observations in mind, we now proceed to a construction of the algebraic solution which will follow closely what has been done in the previous subsection.

We start by expanding the self-consistent propagator $D$ around the free propagator $D_{0}$ and set (see Eq. (II.35)):

$$
D(P)=D_{0}+\delta D=D_{0}(P)+D_{0}(P)(-\Pi) D_{0}(P)+D_{r}(P)
$$

where we have singled out the term linear in $\Pi$, and $D_{r}(P)$ behaves as $\Pi^{2} / P^{6}$ at large $P$, so that the integral $\int_{P} D_{r}(P)$ is finite. Then we generalize the definitions (III.42) and (III.43) and set:

$$
\tilde{\Pi}_{0}=\frac{\Lambda}{2} \int_{P} \delta D(P)
$$

and

$$
\tilde{\Pi}_{2}=\frac{\Lambda}{2} \int_{P} D_{0}(P)+\delta m^{2} .
$$

In these notations, the gap equation reads

$$
\Pi=\tilde{\Pi}_{0}+\tilde{\Pi}_{2},
$$

where only $\tilde{\Pi}_{0}$ depends on the solution $\Pi\left(\tilde{\Pi}_{2}\right.$ is entirely determined by $D_{0}$, as is obvious from Eq. (III.55) and the fact that the counterterms $\delta \lambda$ (in $\Lambda=\lambda+\delta \lambda$ ) and $\delta m^{2}$ are calculated entirely from $D_{0}$ ).

Next, one uses the Bethe-Salpeter equation to eliminate $\Lambda$ in favor of $\Gamma_{0}$ in the defining 
equation for $\tilde{\Pi}_{0}$ (Eq. (III.54) $)$ and get

$$
\begin{aligned}
\tilde{\Pi}_{0} & =\frac{\Lambda}{2} \int_{P} \delta D(P) \\
& =\frac{\Gamma_{0}}{2} \int_{P} \delta D(P)+\frac{\Gamma_{0}}{2} \tilde{\Pi}_{0} \int_{Q} D_{0}^{2}(Q) \\
& =\frac{\Gamma_{0}}{2} \int_{P} D_{r}(P)+\frac{\Gamma_{0}}{2}\left(\tilde{\Pi}_{0}-\Pi\right) \int_{Q} D_{0}^{2}(Q) .
\end{aligned}
$$

At this point we observe that when $\Pi$ is solution of the gap equation (III.56), $\tilde{\Pi}_{0}-$ $\Pi=-\tilde{\Pi}_{2}$, so that the divergence of $\tilde{\Pi}_{0}$ (isolated in the second term of Eq. (III.57) ) is independent of $\Pi$ : this is the main achievement of the elimination of vertex subdivergences.

The gap equation then reads:

$$
\Pi=\frac{\Gamma_{0}}{2} \int_{P} D_{r}(P)+\tilde{\Pi}_{2}\left(1-\frac{\Gamma_{0}}{2} \int_{Q} D_{0}^{2}(Q)\right) .
$$

The first term, which involves $\Pi$ (through $D_{r}$ ) is finite. In the second term, we note that the factor multiplying $\tilde{\Pi}_{2}$ is $\Gamma_{0} / \Lambda$ : this follows from the Bethe-Salpeter with propagators $D_{0}$. Now

$$
\frac{\Gamma_{0}}{\Lambda} \tilde{\Pi}_{2}=\frac{\Gamma_{0}}{\Lambda}\left(\frac{\Lambda}{2} \mathcal{B}_{0}+\delta m^{2}\right)=\frac{\Gamma_{0}}{2} b_{0}\left(m^{2}\right)
$$

where $b_{0}$ is the finite part of $\mathcal{B}_{0}$, and we have set (see Eq. (III.32) )

$$
\delta m^{2}=\frac{\Lambda m^{2}}{32 \pi^{2} \epsilon} .
$$

The gap equation takes then the form:

$$
\begin{aligned}
\Pi & =\frac{\Gamma_{0}}{2} \int_{P} D_{r}(P)+\frac{\Gamma_{0}}{32 \pi^{2}} m^{2}\left(\ln \frac{m^{2}}{\bar{\mu}^{2}}-1\right) \\
& =\frac{\Gamma_{0}}{32 \pi^{2}}\left[\left(m^{2}+\Pi\right) \ln \frac{m^{2}+\Pi}{m^{2}}-\Pi\right]+\frac{\Gamma_{0}}{32 \pi^{2}} m^{2}\left(\ln \frac{m^{2}}{\bar{\mu}^{2}}-1\right)
\end{aligned}
$$

which is now a finite equation. It can also be written in terms of the renormalized coupling constant:

$$
\frac{\Pi}{\lambda}=\frac{m^{2}+\Pi}{32 \pi^{2}}\left(\ln \frac{m^{2}+\Pi}{\bar{\mu}^{2}}-1\right)=\frac{1}{2} b_{0}\left(M^{2}\right),
$$


or in terms of the physical 4-point function $\Gamma$ :

$$
M^{2}=\Gamma\left[\frac{m^{2}}{\lambda}-\frac{M^{2}}{32 \pi^{2}}\right] .
$$

This last form, in which all explicit $\mu$-dependence has disappeared, makes it obvious that $M$ is independent of $\mu$, because $m^{2} / \lambda$ is independent of $\mu$. Indeed, $m_{B}^{2} / \lambda_{B}=$ $\left(m^{2}+\delta m^{2}\right) /\left(\mu^{2 \epsilon} \Lambda\right)$ has a finite limit when $\epsilon \rightarrow 0$, equal to $m^{2} / \lambda$. Since $m_{B}^{2} / \lambda_{B}$ is manifestly independent of $\mu$, so is therefore $m^{2} / \lambda$, which further implies that $m$ runs with $\mu$ in the same way as $\lambda$ does (i.e., according to Eq. (III.50) ).

As made obvious by the previous discussion, the gap equation can be written either in terms of $\Gamma_{0}$ or in terms of $\Gamma$. This reflects a property that we have repeatedly emphasized, namely that the divergent parts of the counterterms do not depend on whether $D$ or $D_{0}$ is used in their calculations. We can exploit this freedom in rearranging the expansion of Eq. (III.53) in the following way:

$$
D(P)=D_{0}+\delta D=D_{0}(P)+D(P)(-\Pi) D(P)+D_{r}^{\prime}(P)
$$

where $D_{r}^{\prime}(P)$ behaves like $1 / P^{6}$, so that the integral $\int_{P} D_{r}^{\prime}(P)$ is finite. Then the full propagator $D$ will naturally appear in the Bethe-Salpeter equation for the vertex counterterms of the gap equation. We can verify that all the steps leading from Eq. (III.53) to the final gap equation (III.63) still hold, with the appropriate replacements of $\Gamma_{0}$ by $\Gamma, D_{0}$ by $D$, and $D_{r}$ by $D_{r}^{\prime}$ (note that $\tilde{\Pi}_{2}$ is not affected). The gap equation (III.63) remains of course unaltered. The advantage of this new expansion becomes apparent when studying the massless limit to which we proceed in the next subsection.

Finally, we note that we could have used a different renormalisation scheme such as the mass-shell renormalization, in which we assume that $m^{2}$ is the physical mass, i.e., $m^{2}=M^{2}$. In this scheme $\Pi=0$, and the mass counterterm is given by

$$
\delta m^{2}=-\frac{\lambda_{B}}{2} \int_{P} \frac{1}{P^{2}+m^{2}}
$$

The coupling constant counterterm can still be chosen as in eq. (111.48), and we have $\Gamma=\Gamma_{0}=\lambda(\bar{\mu}=m)$. This mass-shell scheme, which makes the gap equation trivial at zero temperature, simplifies it at finite temperature, as we shall see in the next subsection. 


\section{E. Finite temperature and massless limit}

The finite-temperature version of the gap equation (III.2) reads:

$$
\Pi=\frac{\Lambda}{2} \mu^{2 \epsilon} \int_{P} D(P)+\frac{\Lambda}{2} \int_{p} \frac{n\left(\varepsilon_{p}\right)}{\varepsilon_{p}}+\delta m^{2},
$$

where $n\left(\varepsilon_{p}\right)=1 /\left(\mathrm{e}^{\beta \varepsilon_{p}}-1\right), \varepsilon_{p}=\sqrt{p^{2}+M^{2}}$ and $\int_{p}$ denotes a 3-dimensional momentum integral. The first integral $\int_{P} D(P)$ is to be understood as before as a $d$-dimensional Euclidean integral, but $D$ now depends on the temperature.

One could naively fear that temperature dependent divergences could appear in the calculation of $\Pi$, as for instance in the second term of Eq. (III.66), due to the presence of the factor $\Lambda$. This however does not happen if one carefully eliminates all the subdivergences which emerge when solving the gap equation iteratively. To understand this, it is enough to consider the first two iterations.

The zeroth iteration is given by Eq. (III.37), with $b_{0}$ replaced by $b_{0}+b_{0}^{T}$. Clearly the countertem (II.27) makes $\Pi^{(0)}$ finite. The first iteration is given by Eq. (III.38), with the finite parts of the integrals including their finite temperature contributions. Potential difficulties could arise for instance from diagram (a) in Fig. 14 in which the temperature dependent piece of the upper loop multiplies the divergent lower loop. But it is easy to verify that this does not happen: indeed the divergence of the lower loop is cancelled by the coupling constant counterterm of diagram (b). Similarly, the contribution proportional to the mass counterterm $\delta m_{0}^{2}$ multiplying the finite temperature part of the loop integral cancels by the same mechanism as at zero temperature. Thus, for the same reason that once subdivergences are eliminated, there remains no finite part coming from some subintegrations multiplying divergent part coming from other subintegrations, there does not remain either finite temperature part multiplying divergent integrals or infinite counterterms. This analysis can be generalized, by going through the same steps as in subsection $\amalg$ IIC, in order to verify that, iteration by iteration, all potentially temperature dependent infinities actually cancel.

To obtain this result in a more explicit fashion, we observe that the term with a statistical factor in Eq. (III.66) can be viewed as a new contribution to $D_{r}$, which is then 
easy to incorporate in the renormalized gap equation (III.62):

$$
\frac{\Pi}{\lambda}=\frac{m^{2}+\Pi}{32 \pi^{2}}\left(\ln \frac{m^{2}+\Pi}{\bar{\mu}^{2}}-1\right)+\frac{1}{2} \int_{p} \frac{n\left(\varepsilon_{p}\right)}{\varepsilon_{p}} .
$$

In the mass-shell renormalization scheme, we get instead

$$
\Pi=\frac{\Gamma_{0}}{2} \int_{P} D_{r}(P)+\frac{\Gamma_{0}}{2} \int_{p} \frac{n\left(\varepsilon_{p}\right)}{\varepsilon_{p}},
$$

which implies that $\Pi \rightarrow 0$ as $T \rightarrow 0$.

Consider now the massless limit $m \rightarrow 0$. In this limit, many of the intermediate manipulations based on the expansion (III.53) of the full propagator $D$ around the free propagator $D_{0}$ become meaningless because of infrared problems. For instance, the BetheSalpeter equation with propagator $D_{0}$, Eq. (III.11), is ill-defined when $m \rightarrow 0$, and therefore so is also the 4 -point function $\Gamma_{0}$. More generally, all the integrals $\mathcal{B}_{k}$ with $k \geq 1$ become infrared divergent when $m=0$. In particular, one cannot take the limit $m \rightarrow 0$ on the gap equation written in the form (III.68) (or (III.61), since both $\Gamma_{0}$ and the integral involving $D_{r}$ develop infrared divergences in this limit. Still, the gap equation has a well defined massless limit, as can be seen by taking this limit at the level of Eq. (III.67) This gives:

$$
\Pi=\frac{\lambda}{32 \pi^{2}} \Pi\left(\ln \frac{\Pi}{\bar{\mu}^{2}}-1\right)+\frac{\lambda}{2} \int_{p} \frac{n\left(\varepsilon_{p}\right)}{\varepsilon_{p}},
$$

which shows that, at finite temperature, the finite self-energy $\Pi$ provides an infrared cutoff, allowing the limit $m \rightarrow 0$ to be taken. This limit can also be written in terms of the full 4-point function $\Gamma$ :

$$
\Pi=-\frac{\Gamma}{32 \pi^{2}} \Pi+\frac{\Gamma}{2} \int_{p} \frac{n\left(\varepsilon_{p}\right)}{\varepsilon_{p}} .
$$

Here, $\Gamma$ is given by Eq. (111.51) with $M^{2}=\Pi$. Thus $\Gamma$ itself depends upon the temperature. From either Eq. (III.69) or Eq. (III.70) one can check that, at zero temperature, $\Pi=0$ in this massless case. As we shall see in Sect. IV, the general approach to renormalization in the massless case is based on the modified expansion in Eq. (III.64), which takes advantage of the presence of the finite-temperature self-energy $\Pi$ as a natural infrared cut-off. 


\section{THE THREE-LOOP SKELETON}

Many of the developments of the previous section can be carried over to the discussion of the three-loop skeleton. In particular, the determination of the coupling constant and mass counterterms will go through the solution of the Bethe-Salpeter equation and the equation for $\partial \Pi / \partial m^{2}$, with little structural changes with respect to the two-loop examples. The essential new aspect of the three-loop example is the presence of the field renormalization constant $\delta Z$, and the fact that the self-energy contributes to the asymptotic behaviour of the propagator.

The three loop skeleton for $\Phi$ is displayed in Fig. 1, and its contribution reads:

$$
\Phi=-\frac{\lambda^{2}}{48} \int_{P} \int_{Q} \int_{R} D(P) D(Q) D(R) D(K+P+Q+R)+\frac{\delta \lambda}{8}\left(\int_{P} D(P)\right)^{2} .
$$

We have added here in $\Phi$ a counterterm which has the structure of the two-loop skeleton; this term is needed to cancel subdivergences, as we shall see. Such a term would be automatically present if we had included in $\Phi$ both the three-loop and the two-loop skeletons. The self-energy follows then from Eq. (I.31):

$$
\Pi(K)=-\frac{\lambda^{2}}{6} \int_{P} \int_{Q} D(P) D(Q) D(K+P+Q)+\frac{\delta \lambda}{2} \int_{P} D(P)+\delta m^{2}+K^{2} \delta Z,
$$

and the kernel of the Bethe-Salpeter equation from Eqs. (II.35):

$$
\Lambda(P, K)=-\lambda^{2} \int_{Q} D(Q) D(K+P+Q)+\delta \lambda
$$

It is important to observe that the vertices of the three-loop contribution in $\Phi$ do not carry coupling constant counterterms. We shall see indeed that, as as we proceed to solving the gap equation through iterations, we do not generate structures which would renormalize these vertices. Such structures would appear only at the 4-loop order in the skeleton expansion. This is a particular illustration of a general feature of $\Phi$-derivable approximations that will be discussed in the next section.

As we indicated in Sect. III, and verified explicitly in the two loop example of Sect. III, the subdivergences associated with vertex and mass renormalizations can be determined 
from the analysis of the Bethe-Salpeter equation and of the equation for the derivative of the self-energy with respect to $m^{2}$. We shall then go through such an analysis. But first we discuss in some details the first orders in perturbation theory obtained from the first two iterations of the gap equation.

\section{A. Perturbation theory}

The zeroth order iteration $\Pi^{(0)}(K)$ is obtained by replacing $D$ by $D_{0}=\left(P^{2}+m^{2}\right)^{-1}$ in the r.h.s. of eq. (IV.2), and coincides with the standard 2-loop contribution of perturbation theory. In dimensional regularization, the divergent part of the diagram is of the form [25]:

$$
\Pi_{\text {div }}^{(0)}(K)=\frac{\lambda^{2}}{\left(16 \pi^{2}\right)^{2}}\left\{\frac{K^{2}}{24 \epsilon}+m^{2}\left[\frac{1}{4 \epsilon^{2}}+\frac{3}{4 \epsilon}+\frac{1}{2 \epsilon} \ln \frac{\bar{\mu}^{2}}{m^{2}}\right]\right\} .
$$

Hidden in these various terms there are three distinct divergences. One is a subdivergence which is removed by a coupling constant counterterm. It will be dealt with shortly. The other two divergences are global divergences corresponding respectively to a mass divergence and a field normalisation divergence. These will be removed by adjusting respectively $\delta m^{2}$ and $\delta Z$. The contribution of $\delta Z$ is easy to identify since it is associated with the unique divergent term proportional to $K^{2}$. Using minimal subtraction we get the following lowest-order contribution $\delta Z_{0}$ to $\delta Z$ :

$$
\delta Z_{0}=-\frac{\lambda^{2}}{\left(16 \pi^{2}\right)^{2}} \frac{1}{24 \epsilon} .
$$

The adjustment of $\delta m^{2}$ can be made only after the proper elimination of the vertex subdivergence. The latter singles out one line in the sunset diagram, namely, that line along which the momentum is kept finite, while the other loop momentum is sent to infinity. In fact, the corresponding subgraph can be identified with $\Lambda_{0}$ (see Fig. 16i), the leading order contribution to $\Lambda$, to within the counterterm $\delta \lambda$; that is:

$$
\Lambda_{0}(P, K)-\delta \lambda=-\frac{\lambda^{2}}{16 \pi^{2} \epsilon}+\frac{\lambda^{2}}{16 \pi^{2}} \int_{0}^{1} \mathrm{~d} x \ln \frac{\left|m^{2}+x(1-x)(P+K)^{2}\right|}{\bar{\mu}^{2}} .
$$




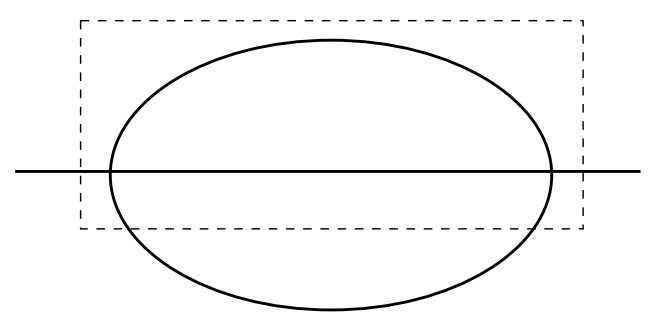

FIG. 16: The dotted line box encloses the subgraph whose divergence is that of the 4-point function $\Lambda_{0}$.

Let us call $\delta \lambda_{0}$ the value of $\delta \lambda$ that renders $\Lambda_{0}$ finite. Minimal subtraction gives:

$$
\delta \lambda_{0}=\frac{\lambda^{2}}{16 \pi^{2} \epsilon}
$$

Note that this contribution, when added to that of Eq. (III.48), reconstructs the full order $\lambda^{2}$ counterterm $\left(3 \lambda^{2} / 32 \pi^{2} \epsilon\right)$. The counterterm (IV.7) generates an order $\lambda^{2}$ contribution to $\Pi$ of the form:

$$
\frac{\delta \lambda_{0}}{2} \mathcal{B}_{0}\left(m^{2}\right)=\frac{\delta \lambda_{0}}{2}\left(\frac{a_{0}}{\epsilon}+b_{0}\right)
$$

which eliminates the subdivergence in $\Pi$, Eq. (IV.4), and the logarithmic term there (via $\left(\delta \lambda_{0} / 2\right) b_{0}$ involving the finite part of $\mathcal{B}_{0}$ in Eq. (IV.8) $)$. There remains then only a global, mass divergence, which is removed by appropriately tuning the counterterm $\delta m^{2}$. Minimal subtraction gives:

$$
\delta m_{0}^{2}=\left(\frac{\lambda m}{32 \pi^{2}}\right)^{2}\left(\frac{1}{\epsilon^{2}}-\frac{1}{\epsilon}\right) .
$$

Note that neither $\delta \lambda_{0}, \delta Z_{0}$ nor $\delta m_{0}^{2} / m^{2}$ depend on $m$ : these counterterms depend only on the asymptotic form of the propagator. Note also that $\delta Z_{0}$ could be obtained by analyzing the divergence which remains after differentiating $\Pi^{(0)}$ with respect to $K^{2}$. Since the coupling constant counterterm is independent of momentum, it does not contribute to the latter derivative, and the same applies to the mass counterterm. This explains why the determination of $\delta Z_{0}$ can be made independently of the elimination of the vertex and mass subdivergences. This property persists in higher orders, as we shall see in Sect. IVC 


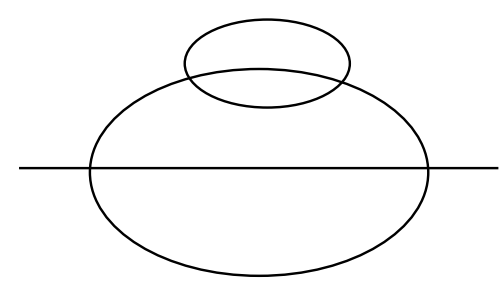

(a)

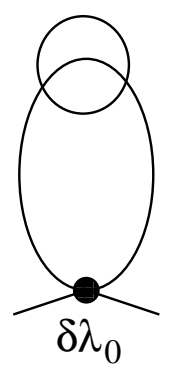

(d)

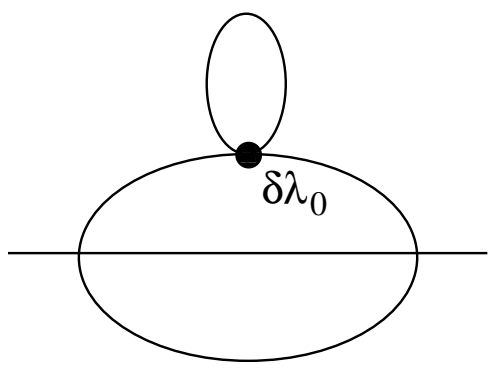

(b)

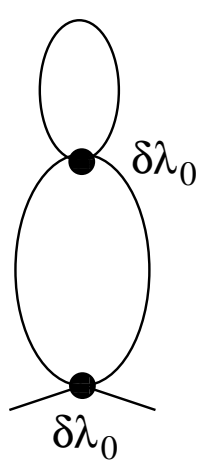

(e)

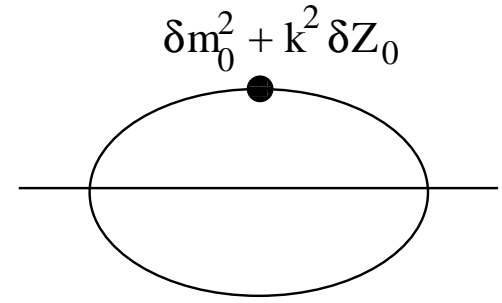

(c)

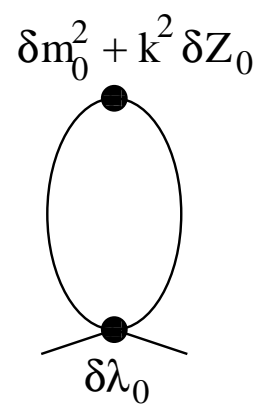

(f)

FIG. 17: The diagrams contributing to $\Pi^{(1)}$, the solution to the gap equation to order $\lambda^{4}$. Diagram (a) is the original diagram with one insertion. All other diagrams involve counterterms which remove the various subdivergences.

For later purposes, we note that the asymptotic behaviour of $\Lambda_{0}$ (after renormalization) is of the form $\Lambda_{0}(P, K) \sim \ln (P+K)^{2} / \mu^{2}$ at large momenta, in agreement with Weinberg's theorem [15, 24] (that $\Lambda_{0}(P, K)$ is a function of $P+K$ can be seen directly on Eqs. (IV.6) or $(\underline{I V .3})$ ). As for $\Pi^{(0)}(K)$, its dominant contribution at large $K$ goes as $K^{2} \ln K^{2}$ (this may be obtained by calculating the finite part of $\Pi^{(0)}(K)$ for $\left.m=0\right)$.

Consider now the first iteration in which we keep only the contributions of order up to $\lambda^{4}$, and call $\Pi^{(1)}$ the corresponding value of $\Pi$. The associated diagrams are shown in Fig. 17. Not represented there are the diagrams involving the new counterterms that need to be adjusted at this iteration, namely, $\delta \lambda_{1}, \delta m_{1}^{2}$, and $\delta Z_{1}$.

Let us focus on the new vertex subdivergences. The corresponding diagrams are displayed in Fig. 18, The subgraph within the box in Fig. 18] a is logarithmically divergent 
because the inserted self-energy grows like $K^{2} \ln K^{2}$ at large $K$, as mentioned earlier; thus, this insertion does not change the degree of divergence of the diagram, the factor $K^{2} \ln K^{2}$ cancelling the $1 / K^{2}$ coming from the extra propagator. The subdivergence in Fig. 18, a (together with the corresponding counterterms from Figs. 17]b and 17. c) can be recognized as the contribution of order $\lambda^{4}$ to the (global) divergence of the integral in Eq. (IV.3) for the kernel $\Lambda$ of the Bethe-Salpeter equation. In other terms, the subgraph singled out in Fig. 18], represents the first correction to the zeroth order kernel $\Lambda_{0}$ in Eq. (IV.6), and its divergence can be absorbed in a correction $\delta \lambda_{0}^{(1)}$ of order $\lambda^{4}$ to $\delta \lambda_{0}$. This type of correction is a new feature which did not appear in the two-loop example: it is related to the fact that the insertion of a self-energy affects the asymptotic behaviour of the propagator. However, the other 4-point divergence, corresponding to the subgraph in

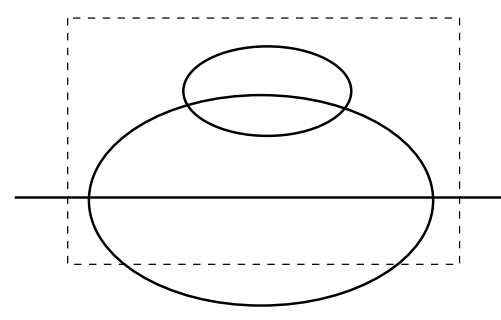

(a)

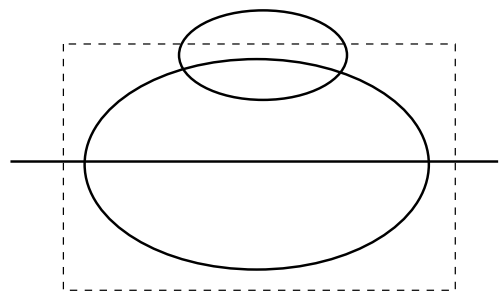

(b)

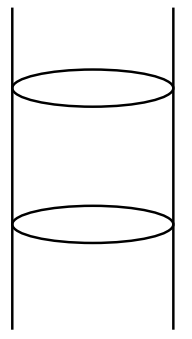

(c)

FIG. 18: Vertex subdivergences in the diagrams contributing to $\Pi^{(1)}$. The subdivergence represented by the dashed-line box in figure (b) is that of the 4-point function depicted in figure (c), and is removed by a counterterm determined by the Bethe-Salpeter equation.

the box in Fig. 18, b is analogous to those encountered in the two-loop example. It is the divergence of the 4-point function shown in Fig. 18. c, obtained as the first iteration of the Bethe-Salpeter equation with the leading order kernel $\Lambda_{0}$. The corresponding divergence is absorbed in the counterterm $\delta \lambda_{1}$ (after subdivergences are eliminated by counterterms such as those in Figs. 17]b, d and e).

To summarize, at the first iteration, and order $\lambda^{4}$, the vertex counterterm receives two contributions: one, which we call $\delta \lambda_{0}^{(1)}$, removes the order- $\lambda^{4}$ divergence from the kernel 
$\Lambda$, the other, which we call $\delta \lambda_{1}$, renormalizes the first iteration of the Bethe-Salpeter equation. Once the vertex subdivergences are removed, the mass counterterm $\delta m_{1}^{2}$ can be chosen so as to remove the remaining mass divergences from $\Pi^{(1)}$. Finally, $\delta Z_{1}$ can be

obtained by analyzing the divergence which remains after differentiating $\Pi^{(1)}$ with respect to $K^{2}$. The same argument that we used at leading order shows that this can be computed independently of the vertex and mass renormalizations, that is, $\delta Z_{1}$ can be obtained by extracting the divergence proportional to $K^{2}$ in the sum of the two diagrams (a) and (c) of Fig. 177 (with only $K^{2} \delta Z_{0}$ in diagam (c)).

In order to obtain a more systematic determination of the coupling constant and mass counterterms, we turn now to the analysis of the Bethe-Salpeter equation and the equation for $\partial \Pi / \partial m^{2}$. We shall return to $\delta Z$ in Sec. IVC

\section{B. The Bethe-Salpeter equation}

As we did in the previous section, we shall consider the solution obtained by iterations, using first in the integral of Eq. (II.17) the free propagator $D_{0}$. We shall, as before, call $\Gamma_{0}$ the corresponding solution, $\Lambda_{0}$ the associated kernel, and construct $\Gamma_{0}$ as a formal series by solving the Bethe-Salpeter equation by iterations. We have:

$$
\begin{aligned}
\Gamma_{0}(P, K) & =\Lambda_{0}(P, K)-\frac{1}{2} \int_{Q} \Lambda_{0}(P, Q) D_{0}^{2}(Q) \Gamma_{0}(Q, K) \\
& =\Lambda_{0}(P, K)-\frac{1}{2} \int_{Q} \Gamma_{0}(P, Q) D_{0}^{2}(Q) \Lambda_{0}(Q, K) .
\end{aligned}
$$

In leading order, and before renormalization, $\Gamma_{0}^{(0)}$ is equal to $\Lambda_{0}$, which is divergent. This divergence has been dealt with in Sect. IVA where we have determined the counterterm $\delta \lambda_{0}$ needed to absorb it $\left(\delta \lambda_{0}\right.$ is given by Eq. (IV.7)). We shall call $\tilde{\Lambda}_{0}$ the finite quantity

$$
\tilde{\Lambda}_{0}(P, K) \equiv \delta \lambda_{0}-\lambda^{2} \int_{Q} D_{0}(Q) D_{0}(K+P+Q) .
$$

Thus, after renormalization, $\Gamma_{0}^{(0)}=\tilde{\Lambda}_{0}$. The quantities $\tilde{\Lambda}_{0}$ and $\Lambda_{0}$ differ by the counterterm difference $\delta \lambda-\delta \lambda_{0}$ which will be needed to remove further divergences appearing as we 
proceed with the iterative solution of Eq. (IV.10).

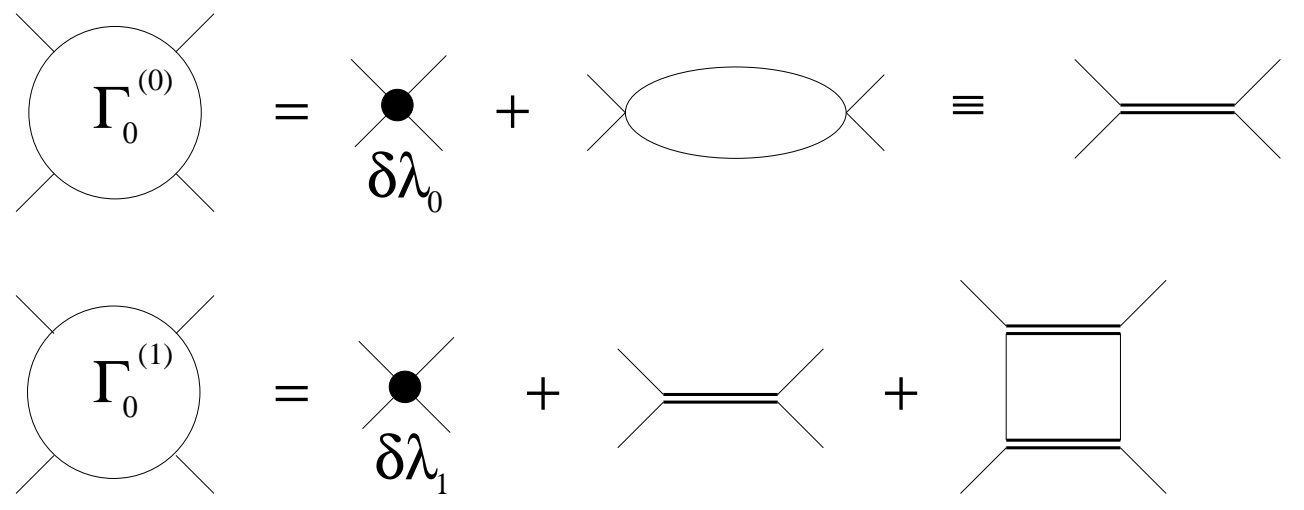

FIG. 19: The contributions to $\Gamma_{0}$ obtained from the zeroth and first iteration of the BetheSalpeter equation. The horizontal double bar represents $\tilde{\Lambda}_{0}$. The counterterm $\delta \lambda_{1}$ absorbs the divergence of the loop integral in the last diagram for $\Gamma_{0}^{(1)}$ (see Eq. (IV.12)).

The first iteration reads

$$
\Gamma_{0}^{(1)}(P, K)=\tilde{\Lambda}_{0}(P, K)+\delta \lambda_{1}-\frac{1}{2} \int_{Q} \tilde{\Lambda}_{0}(P, Q) D_{0}^{2}(Q) \tilde{\Lambda}_{0}(Q, K) .
$$

The right hand side contains no subdivergences: these are eliminated by the counterterm which makes $\tilde{\Lambda}_{0}$ finite. What remains in the integral is then a global divergence which can be absorbed in $\delta \lambda_{1}$. That the divergence of the integral in Eq. (IV.12) is a local one can be easily verified by observing that the derivative of this integral with respect to $P_{\mu}$ leaves a finite integral. This can be checked by using the explicit form of $\tilde{\Lambda}_{0}(P, K)$ given in Eq. (IV.11). This implies that the counterterm cannot depend on $P$. For the same reason, it cannot depend on $K$. Hence it is a constant, which can be chosen by minimal subtraction, as we did earlier for $\delta \lambda_{0}$, or by imposing a renomalization condition on $\Gamma_{0}(P, K)$ at some values of the momenta. We shall implicitly continue using minimal subtraction. A diagrammatic interpretation of the first two iterations is given in Fig. 19, 
It is instructive to perform another iteration and get:

$$
\begin{aligned}
\Gamma_{0}^{(2)}(P, K) & =\tilde{\Lambda}_{0}(P, K)+\delta \lambda_{1}+\delta \lambda_{2} \\
& -\frac{1}{2} \int_{Q}\left[\tilde{\Lambda}_{0}(P, Q)+\delta \lambda_{1}\right] D_{0}^{2}(Q)\left[\tilde{\Lambda}_{0}(Q, K)+\delta \lambda_{1}\right] \\
& +\frac{1}{4} \int_{Q} \int_{R} \tilde{\Lambda}_{0}(P, Q) D_{0}^{2}(Q) \tilde{\Lambda}_{0}(Q, R) D_{0}^{2}(R) \tilde{\Lambda}_{0}(R, K),
\end{aligned}
$$

where we have dropped terms of order $\lambda^{8}$ or higher. The contributions of order $\lambda^{6}$ are depicted in Fig. 20, One can then again proceed to an analysis of the subdivergences. Note that there are no subdivergence involving only one line of a $\Lambda_{0}$ (since opening one line of a $\Lambda_{0}$ would lead to a convergent 6-point function); in other words the divergent subdiagrams contain complete $\Lambda_{0}$ as subdiagrams [17]. Again, one finds that all the subdivergences are eliminated by the counterterms determined at the previous iteration, namely $\delta \lambda_{0}$ and $\delta \lambda_{1}$. The remaining global divergence is absorbed in the new local counterterm $\delta \lambda_{2}$. That this divergence is a local one can be verified by the same argument as given after Eq. (IV.12), namely by differentiating with respect to the external momenta and verifying that all the resulting contributions are finite.

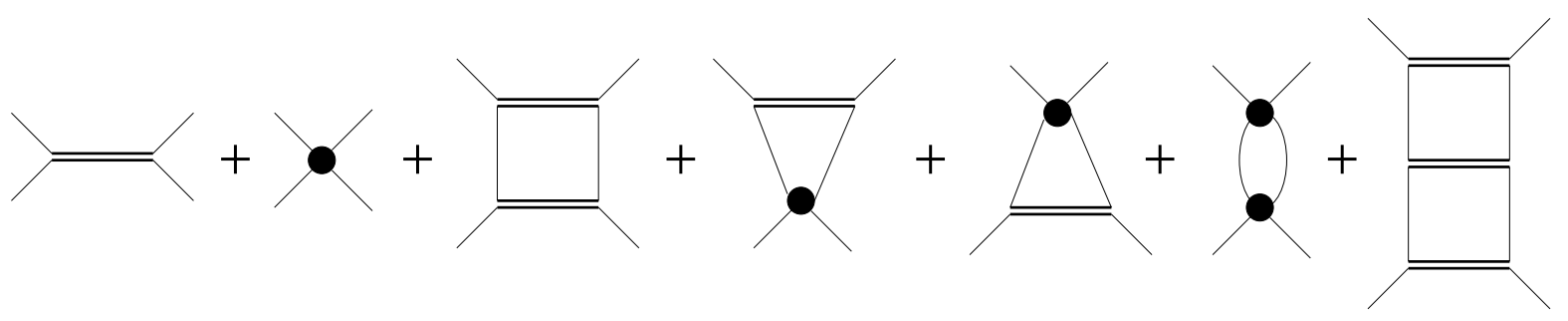

FIG. 20: The contributions of order $\lambda^{6}$ to $\Gamma_{0}^{(2)}$ obtained from the second iteration of the BetheSalpeter equation, illustrating the elimination of the subdivergences leading to the determination of the global counterterm $\delta \lambda_{2}$. The kernel $\tilde{\Lambda}_{0}$ (which includes its counterterm $\delta \lambda_{0}$ ) is represented by a horizontal bar, as in Fig. 19. The black dots represents the counterterm $\delta \lambda_{1}$, except for the first one on the left which is the sum of $\delta \lambda_{1}$ and $\delta \lambda_{2}$.

It is clear that the successive iterations of the Bethe-Salpeter equation will reproduce a structure analogous to that obtained in Sect. IIIC for the two-loop skeleton, with the 
contribution $\Gamma_{0}^{(n)}$ at iteration $n$ being of order $\lambda^{2 n+2}$. In particular, the contributions involving the product of a counterterm $\delta \lambda_{k}$ by a finite integral cancel out (such terms appear for instance in the second line of Eq. (IV.13) and compensate contributions of the first line where one of the integration variables is kept fixed while the other one is taken to infinity). This is the kind of cancellations expected in the BPH analysis [15], and guarantees, in particular, that when the calculation is done at finite temperature, there does not remain, at any iteration, terms involving the product of an infinite counterterm by a finite temperature contribution.

The only significant difference with the 2-loop example discussed in the previous section, is that the divergences do not simply factorize because the kernel is momentum dependent, and no simple expression can be given for the counterterms. Because of this, one may find useful to have finite equations for $\Gamma$ in which those counterterms do not explicitly enter. Consider for instance $\Gamma_{0}(0, P)$. This can be obtained from the following finite equation:

$$
\begin{aligned}
\Gamma_{0}(0, P)-\Gamma_{0}(0,0)= & \Lambda_{0}(0, P)-\Lambda_{0}(0,0) \\
& -\frac{1}{2} \int_{Q} \Gamma_{0}(0, Q) D_{0}^{2}(Q)\left\{\Lambda_{0}(Q, P)-\Lambda_{0}(Q, 0)\right\} .
\end{aligned}
$$

It is easily verified that $\Lambda_{0}(Q, P)-\Lambda_{0}(Q, 0) \sim P / Q$ for $Q^{2} \gg P^{2}$. Since $\Gamma_{0}(0, Q)$ grows at most logarithmically (this follows from Weinberg's theorem [15], 24]), the integral over $Q$ in Eq. (IV.14) is indeed finite. Thus, Eq. (IV.14) is finite, and can be solved to determine $\Gamma_{0}(P, 0)$ in terms of $\Gamma_{0}(0,0)$, which is supposed to be finite and may be chosen to satisfy a renormalization condition. Once $\Gamma_{0}(0, P)$ is known, it may be used to determine the full momentum dependence of $\Gamma_{0}(K, P)$. The difference $\Gamma_{0}(K, P)-\Gamma_{0}(0, P)$ is indeed given by the finite equation

$$
\begin{aligned}
\Gamma_{0}(K, P)-\Gamma_{0}(0, P)= & \Lambda_{0}(K, P)-\Lambda_{0}(0, P) \\
& -\frac{1}{2} \int_{Q}\left\{\Lambda_{0}(K, Q)-\Lambda_{0}(0, Q)\right\} D_{0}^{2}(Q) \Gamma_{0}(Q, P) .
\end{aligned}
$$


By combining Eqs. (IV.14) and (IV.15), one gets also:

$$
\begin{aligned}
\Gamma_{0}(K, P)-\Gamma_{0}(0,0)= & \Lambda_{0}(K, P)-\Lambda_{0}(0,0) \\
& -\frac{1}{2} \int_{Q}\left\{\Lambda_{0}(K, Q)-\Lambda_{0}(0, Q)\right\} D_{0}^{2}(Q) \Gamma_{0}(Q, P) \\
& -\frac{1}{2} \int_{Q} \Gamma_{0}(0, Q) D_{0}^{2}(Q)\left\{\Lambda_{0}(Q, P)-\Lambda_{0}(Q, 0)\right\},
\end{aligned}
$$

an equation similar to that first obtained by van Hees and Knoll [17].

At this point, we note that the previous analysis departs somewhat from strict perturbation theory: we have indeed left out at each iteration perturbative contributions which correspond to self-energy insertions in the propagators of the Bethe-Salpeter equation, or in the lines of $\Lambda_{0}$. Let us then consider the effect of such self-energy insertions. We assume that the added self-energy contains all the counterterms needed to make it finite. The question arises then of whether this insertion brings in new types of subdivergences. The answer is clearly negative. Indeed, a new subdivergence could be obtained only by keeping finite the momentum in one of the internal line of the added self-energy. This is equivalent to opening that line, leading to a convergent 6-point function. It follows that the structure of subdivergences exhibited in the previous analysis is not modified by the replacement of $D_{0}$ by another propagator $D$. However, contrary to what happens in the case of the 2-loop skeleton where a similar insertion of a self-energy on the intermediate propagator of the Bethe-Salpeter equation only affects the finite part of $\Gamma$, here the divergent part is also modified. This is because the dominant asymptotic behaviour of $D$ is altered when the two-loop self-energy is included in the propagator. This implies that the numerical values of the counterterms obtained at a given iteration of the Bethe-Salpeter equation, such as $\delta \lambda_{0}$ in Eq. (IV.11), or $\delta \lambda_{1}$ in Eq. (IV.12), will receive corrections to all orders in the coupling constant coming from successive insertions of the type we have just discussed. We have already met an example of such a correction in the previous subsection, namely the correction $\delta \lambda_{0}^{(1)}$ to $\delta \lambda_{0}$ (cf. Fig. [18, a).

It is then useful here to return to the gap equation in order to see diagrammatically how these various corrections show up as we proceed through the iterations of this equation. For illustration, some typical diagrams contributing to $\Pi^{(2)}$ at order $\lambda^{6}$ are displayed in 
Fig. 21. The vertex subdivergences are the global divergences of the 4-point subgraphs enclosed within the dashed line boxes. Those in Figs. 21 a-c correspond to a renormalization of the kernel $\Lambda$ of the Bethe-Salpeter equation: they can be absorbed in a correction $\delta \lambda_{0}^{(2)}$ of order $\lambda^{6}$ to $\delta \lambda_{0}$. The two boxes in Figs. 211 d and e correspond to propagator corrections on the first iteration of the Bethe-Salpeter equation: they contribute to the correction $\delta \lambda_{1}^{(1)}$ of $\delta \lambda_{1}$ (compare to Fig. [18,b and c). Finally, the subdivergence in Fig. 21] $\mathrm{f}$ corresponds to the second iteration of the Bethe-Salpeter equation: the corresponding divergence is aborbed into $\delta \lambda_{2}$ (after proper elimination of the subdivergences).

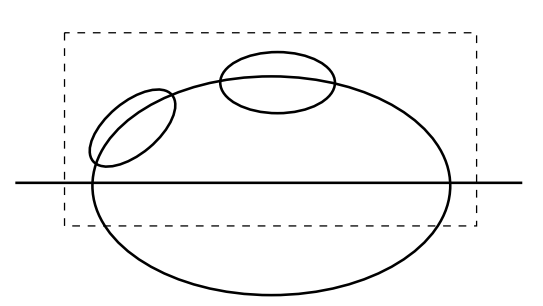

(a)

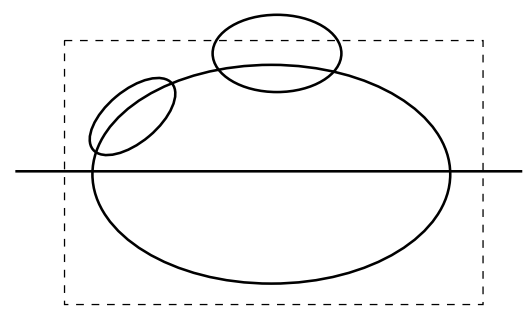

(d)

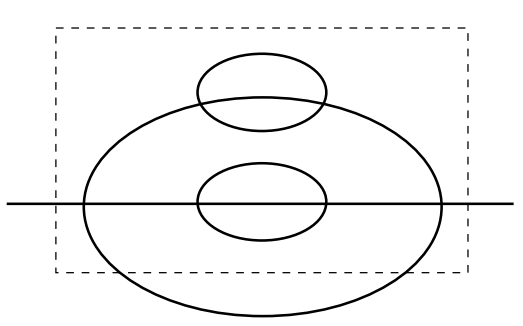

(b)

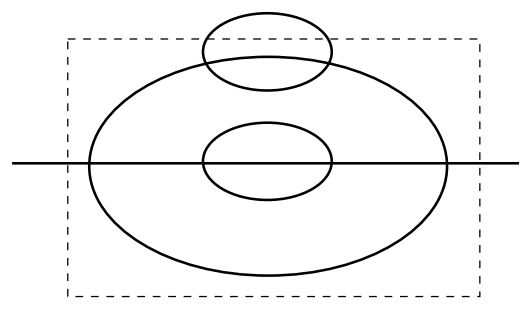

(e)

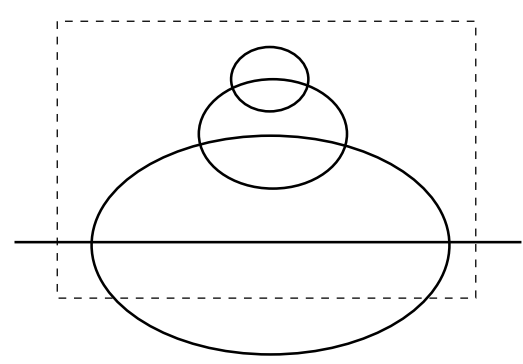

(c)

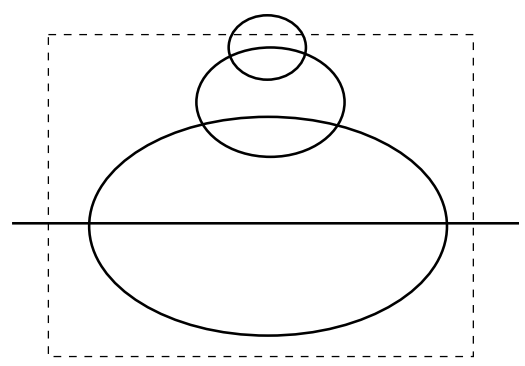

(f)

FIG. 21: Diagrams illustrating some of the new subdivergences which occur at order $\lambda^{6}$. The divergent subgraphs are enclosed in the dashed line boxes.

We turn now to the mass counterterm $\delta m^{2}$. As we have seen, this can be deduced from the equation obtained by differentiating the gap equation with respect to $m^{2}$. We can rewrite Eq. (11.36) as follows:

$$
\int_{Q}\left[\delta(K-Q)+\frac{1}{2} \Lambda(K, Q) D^{2}(Q)\right] \frac{\partial \Pi(Q)}{\partial m^{2}}=-\frac{1}{2} \int_{Q} \Lambda(K, Q) D^{2}(Q)+\frac{\delta m^{2}}{m^{2}} .
$$


This equation has a simple diagrammatic interpretation similar to that given in Fig. 11, with now $\Lambda$ replacing the coupling constant of Fig. 11. The diagrams contributing to $\partial \Pi / \partial m^{2}$ that are generated by this equation have vertex and mass subdivergences, analogous to those of the self-energy itself. It is however easy to verify that the vertex subdivergences are eliminated by the same counterterms as those which make finite the Bethe-Salpeter equation. This is most readily seen on the following form of the equation:

$$
\frac{\partial \Pi(P)}{\partial m^{2}}=-\frac{1}{2} \int_{Q} \Gamma(P, Q) D^{2}(Q)+\frac{\delta m^{2}}{m^{2}} \int_{K}\left[\delta(P-K)-\frac{1}{2} \Gamma(P, K) D^{2}(K)\right],
$$

obtained from Eq. (IV.17) by using Eqs. (II.21)-(II.22). This expression of $\partial \Pi / \partial m^{2}$ in terms of $\Gamma$ contains no vertex subdivergences. The remaining divergences can be absorbed in the mass counterterm, determined then by requiring that $\partial \Pi / \partial m^{2}$ be finite. We shall illustrate this cancellation of subdivergences, by looking at a few iterations of Eq. (IV.18). For the sake of simplicity, we use in all expressions the free propagator $D_{0}$, rather than the full propagator $D$ (as we have already mentioned, this does not change the structure of the counterterms, only their numerical values; in particular the way the various subdivergences cancel is independent of this choice). In zeroth order we have

$$
\frac{\delta m_{0}^{2}}{m^{2}}=\left[\frac{1}{2} \int_{Q} \tilde{\Lambda}_{0}(P, Q) D_{0}^{2}(Q)\right]_{\mathrm{div}}
$$

where the symbol $[\cdots]_{\text {div }}$ isolates the divergent part of the quantities inside the brakets. One can verify that Eq. (IV.19) leads to the expression (IV.9) of the mass counterterm obtained in perturbation theory. The first iteration gives

$$
\frac{\delta m_{1}^{2}}{m^{2}}=-\frac{1}{2}\left[\int_{Q} \Gamma_{0}^{(1)}(P, Q) D^{2}(Q)\right]_{\mathrm{div}}+\frac{\delta m_{0}^{2}}{m^{2}} \int_{K}\left(\delta(P-K)-\frac{1}{2} \tilde{\Lambda}_{0}(P, K) D_{0}^{2}(K)\right),
$$

which after using Eq. (IV.12) leads to

$$
\begin{aligned}
\frac{\delta m_{1}^{2}}{m^{2}} & =\frac{1}{2}\left[\int_{Q}\left(\delta \lambda_{1}+\frac{1}{2} \int_{K} \tilde{\Lambda}_{0}(P, K) D_{0}^{2}(K) \tilde{\Lambda}_{0}(K, Q)\right) D_{0}^{2}(Q)\right]_{\mathrm{div}} \\
& +\frac{\delta m_{0}^{2}}{m^{2}} \int_{K} \frac{1}{2} \tilde{\Lambda}_{0}(P, K) D_{0}^{2}(K) .
\end{aligned}
$$

In contrast to the case of the 2-loop skeleton, where the multiple integrals factorize, here one cannot obtain an explicit expression. One can however easily recognize the role of 
counterterms determined at a previous iteration (here $\delta m_{0}^{2}$ ), or those coming from the Bethe-Salpeter equation (here $\delta \lambda_{1}$ ), in the cancellation of subdivergences. Observe in particular that the product of $\delta m_{0}^{2}$ by the finite part of the integral in the second line, which is $P$-dependent, cancels with a corresponding term in the first line which arises by keeping $K$ fixed and taking $Q$ to infinity.

\section{The determination of $\delta Z$ and the asymptotic form of $\Pi$}

In the discussion of the two-loop approximation in Sect. II, we have been able to separate the calculation of the vertex and mass counterterms $\delta \lambda$ and $\delta m^{2}$ from the resolution of the gap equation for the propagator. This has been possible since the divergences appearing in the Bethe-Salpeter equation for $\Gamma$, and also in Eq. (III.25) for $\partial \Pi / \partial m^{2}$, depend only upon the leading behaviour of the propagator at large momenta, and this is not modified by the resummation performed by the one-loop gap equation (III.2). Therefore, in order to compute the counterterms $\delta \lambda$ and $\delta m^{2}$, it was enough to consider these equations with the full propagator replaced by the free one, $D_{0}(P)=\left(P^{2}+m^{2}\right)^{-1}$ (where the mass $m$ did not play any role except that of an infrared regulator).

But in the present, three-loop approximation, such simplifications no longer hold. Indeed, as revealed by the analysis of the first orders of perturbation theory, the insertion of the self-energy does change the asymptotic behavior of the propagator, so that this behavior keeps changing as one proceeds through the iterations. Thus, if one were to compute the propagator and the various counterterms through iterations, both the gap equation and the equations for $\delta \lambda$ and $\delta m^{2}$ would have to be solved simultaneously. Still, as we explain now, it is nevertheless possible to disentangle the calculation of the counterterms from that of the full solution to the gap equation. The key observation is that the counterterms are sensitive only to the leading asymptotic behavior of the propagator. This is clear in the previous perturbative analysis, and in the general case it results from the fact that Eqs. (II.17) and (IV.18) involve only logarithmic divergences. As for $\delta Z$, it is determined by the global divergence of the self-energy diagrams obtained when all the 
internal loop momenta are simultaneously sent to infinity (after all subdivergences have been removed): this divergence is proportional to $K^{2}$, and is independent of the mass, or any other subleading contribution to the propagator at large momenta.

Now, according to Weinberg's theorem [15, 24], one expects that for very large momenta $K \gg m, \Pi(K) / K^{2}$ grows, at most, as a (slowly varying) function of $\ln K^{2}$. It is useful to introduce the special notation $\Pi_{2}(K)$ for the leading asymptotic contribution: by assumption $\left(\Pi-\Pi_{2}\right) / K^{2} \rightarrow 0$ as $K^{2} \rightarrow \infty$. To compute $\Pi_{2}(K)$, we need the limit of Eq. (IV.2) for very high $K^{2}$ (and zero temperature). Since the mass plays no role in this limit, one could set $m=0$, and we shall indeed do that eventually. However in practice, it is sometimes useful to keep a non-zero mass to avoid infrared complications, so let us consider first the case $m \neq 0$. The corresponding gap equation involves all types of divergences, but, for the purpose of computing $\Pi_{2}$, it can here be renormalized by simple subtractions. That is, $\Pi_{2}(K)$ can be obtained from the large $K$ behavior of the solution $\Pi$ to the following, auxiliary, gap equation:

$$
\Pi(K)=S\left(K^{2}\right)-S\left(K^{2}=-m^{2}\right)-\left.\left(K^{2}+m^{2}\right) \frac{\partial S}{\partial K^{2}}\right|_{K^{2}=-m^{2}} .
$$

where $S(K)$ denotes the r.h.s. of Eq. (IV.2). This equation has been used in Ref. [18] to compute the self-energy in the vacuum. It is finite indeed, as it can be checked by taking two derivatives with respect to $K^{2}$ within the integrand, and noticing that the result is finite. It should be emphasized that this procedure holds because, in the present approximation, the vertex counterterm $\delta \lambda$ enters only a local self-energy diagram (the tadpole in the r.h.s. of Eq. (IV.2) ), and thus it can be simulated by a local, mass, subtraction. In any case, Eq. (IV.22) is correct only for the calculation of $\Pi_{2}$, and not for the subleading contribution $\Pi_{0} \equiv \Pi-\Pi_{2}$.

In what follows we shall compute $\Pi_{2}$ from the gap equation with $m=0$, using dimensional regularization. Then, both the mass divergences and the vertex subdivergences automatically cancel out, and the remaining field divergences can be eliminated by the counterterm $\delta Z K^{2}$. The equation determining $\Pi_{2}$ reads then

$$
\Pi_{2}(K)=S(K)+K^{2} \delta Z
$$


where this time $S(K)$ denotes the r.h.s. of Eq. (IV.2) evaluated with propagator $D_{2}^{-1}(K) \equiv K^{2}+\Pi_{2}(K)$. Clearly, the finite form of the equation, and thus also the ensuing solution for $\Pi_{2}$, will depend upon our choice of a subtraction scheme. For instance, one can choose $\delta Z=-\left.\left(\partial S / \partial K^{2}\right)\right|_{K^{2}=-\mu^{2}}$, in which case the asymptotic behaviour of the propagator will depend upon the subtraction scale $\mu$. Since, as we have seen before, the counterterms $\delta \lambda$ and $\delta m^{2}$ (including their divergent parts) are sensitive to the asymptotic behavior of the propagator, it follows that these counterterms will end up being dependent upon the renormalization scheme for $\delta Z$.

We can now summarize the analysis of the last two subsections as follows: i) The values of the counterterms are determined by the dominant piece of the self-energy at large $K$. ii) They can therefore be determined from the solution of an auxiliary gap equation which coincides at asymptotically large $K$ with the original gap equation. iii) The coupling renormalization involves two types of counterterms: those which renormalize the kernel $\Lambda$ of the Bethe-Salpeter equation, and those which render finite the iterations of the Bethe-Salpeter equation. The counterterms of the first kind can be summed up as the counterterm $\delta \lambda_{0}$. The counterterms of the second kind can be computed in terms of the solution to the Bethe-Salpeter equation. iv) The mass counterterms are determined by the equation for $\partial \Pi / \partial m^{2}$ where the vertex subdivergences are eliminated by the counterterms which render finite the Bethe-Salpeter equation.

\section{The gap equation: algebraic renormalization}

The verification that, with the counterterms determined as indicated above, the gap equation is indeed finite, goes through similar steps as in subsection [IID The main complication here comes from the need to deal with the modification of the asymptotic part of the propagator resulting from the insertion of the momentum dependent selfenergy. We shall handle this by considering an auxiliary gap equation, as indicated in the previous subsection. 
We write the full propagator as

$$
D(K)=\frac{1}{K^{2}+m^{2}+\Pi(K)}
$$

where $\Pi(K)$ is the renormalized self-energy. Then we set

$$
\Pi=\Pi_{0}+\Pi_{2}
$$

where $\Pi_{2}$ is the solution of an auxiliary gap equation which coincides asymptotically with the original gap equation. As indicated in the previous subsection, $\Pi_{2}$ may be obtained, for instance, as the solution of the gap equation with $m=0$, that is:

$$
\Pi_{2}(K)=-\frac{\lambda^{2}}{6} \int_{P} \int_{Q} D_{20}(P) D_{20}(Q) D_{20}(K+P+Q)+K^{2} \delta Z,
$$

where $D_{20}(P)^{-1} \equiv P^{2}+\Pi_{2}(P)$. Note that, thus defined, $\Pi_{2}(0)=0$. We denote by $\Pi_{0}(K)$ the difference between the true solution, $\Pi$, and $\Pi_{2}$. It follows from our assumptions that:

$$
\lim _{K \rightarrow \infty} \frac{\Pi_{0}(K)}{K^{2}}=0,
$$

so that $\Pi_{0}(K)$ grows at most logarithmically at large $K$. There is of course some arbitrariness in the decomposition (IV.25), related to the specific choice one makes for $\Pi_{2}$. The important point is that the asymptotic behaviour of $\Pi_{2}(K)$ at large $K$ coincides with that of $\Pi(K)$. Indeed, from the previous discussions, we know that the counterterms depend only on $\Pi_{2}$ and are insensitive to the precise separation between $\Pi_{2}$ and $\Pi_{0}$.

The propagator

$$
D_{2}(K) \equiv\left(K^{2}+m^{2}+\Pi_{2}\right)^{-1}
$$

will play the same role here as $D_{0}(K)$ in the previous section, i.e., we shall expand with respect to it. We include $m^{2}$ within $D_{2}$ in order to avoid possible infrared problems in this expansion (the massless case is discussed in Sec. IVF). We call $\Gamma_{2}$ the solution of the Bethe-Salpeter equation where the intermediate propagators are $D_{2}$ propagators and similarly the kernel $\Lambda_{2}$ is evaluated with $D_{2}$ propagators. We shall assume that the counterterms $\delta \lambda$ and $\delta m^{2}$ are determined from this equation and the corresponding 
one for $\partial \Pi / \partial m^{2}$. That is, the solution of the auxiliary gap equation provides a finite $\Pi_{2}$ and well defined values for the counterterms $\delta Z, \delta \lambda$ and $\delta m^{2}$. We verify now that these counterterms render the initial gap equation finite. The strategy will be to use the auxiliary gap equation to obtain $D_{2}$ and then determine the counterterms. These counterterms will then be used in order to eliminate the divergences of the gap equation, leading to a finite equation for $\Pi_{0}$.

It is useful first to introduce a special notation for the self-energy calculated with the propagator $D_{2}$. We set $\tilde{\Pi}_{2}(K)=\Pi\left[D_{2}\right]$, where $\Pi[D]$ denotes the right hand side of Eq. (IV.2); that is:

$$
\tilde{\Pi}_{2}(K)=-\frac{\lambda^{2}}{6} \int_{P} \int_{Q} D_{2}(P) D_{2}(Q) D_{2}(K+P+Q)+\frac{\delta \lambda}{2} \int_{P} D_{2}(P)+\delta m^{2}+K^{2} \delta Z .
$$

Note that if the counterterms $\delta \lambda$ and $\delta m^{2}$ in this equation are limited to their leading order values, $\delta \lambda_{0}$ and $\delta m_{0}^{2}$ respectively (calculated with propagator $D_{2}$ ), then $\tilde{\Pi}_{2}(K)$ is finite.

We now expand the propagator $D$ around $D_{2}$

$$
D=D_{2}+\delta D \quad \delta D=D_{2}\left[-\Pi_{0}\right] D_{2}+D_{r}
$$

where we have singled out in $\delta D$ the term linear in $\Pi_{0}$. The usefulness of this expansion is that each extra power of $\Pi_{0}$ decreases the degree of divergence of the diagram in which the corresponding propagator is inserted. This property will allow us to isolate the vertex subdivergences in very much the same way as in the two-loop example. In analogy to what we did in Sect. IIID we define

$$
\tilde{\Pi}_{0}(K)=\frac{1}{2} \int_{P} \Lambda_{2}(K, P) \delta D(P)
$$

Now we note that the gap equation can be written as $\Pi=\Pi[D]$ where $D$ is expanded as in Eq. (IV.30), and may thus be considered a function of $\Pi_{0}$. Using the definitions (IV.29) and (IV.31) above, we can put the gap equation in the form

$$
\Pi=\tilde{\Pi}_{2}+\tilde{\Pi}_{0}+\tilde{\Pi}_{r}
$$


where $\Pi$ in the left hand side is to be understood as $\Pi_{2}+\Pi_{0}$ (so that the equation is efffectively an equation for $\left.\Pi_{0}\right)$. The quantity $\tilde{\Pi}_{r}(K)$ is not only finite, but decreases as $1 / K^{2}$ at large $K$. This follows from Weinberg's theorem and the fact that $\tilde{\Pi}_{r}$ contains at least two $\Pi_{0}$ insertions and is therefore of degree of divergence -2 .

Next we note that

$$
\tilde{\Pi}_{0}(K)-\tilde{\Pi}_{0}(0)=\frac{1}{2} \int_{P}\left[\Lambda_{2}(K, P)-\Lambda_{2}(0, P)\right] \delta D(P)
$$

is finite (cf. after Eq. (IV.14) $)$. Since both $\tilde{\Pi}_{0}(K)-\tilde{\Pi}_{0}(0)$ and $\tilde{\Pi}_{2}(K)-\tilde{\Pi}_{2}(0)$ are finite, so is therefore $\Pi(K)-\Pi(0)$. It follows that the ultraviolet divergences are entirely contained in $\Pi(0)=\tilde{\Pi}_{2}(0)+\tilde{\Pi}_{0}(0)+\tilde{\Pi}_{r}(0)$ where both $\tilde{\Pi}_{2}(0)$ and $\tilde{\Pi}_{0}(0)$ are a priori divergent.

For $\tilde{\Pi}_{0}(0)$ we proceed as in Sect. IIID and express $\Lambda_{2}(0, P)$ in the defining equation for $\tilde{\Pi}_{0}(0)$ (Eq. (IV.31) with $K=0$ ) in terms of $\Gamma_{2}(0, P)$ (the solution to the Bethe-Salpeter equation with propagator $D_{2}$ ). We get:

$$
\begin{aligned}
\tilde{\Pi}_{0}(0) & =\frac{1}{2} \int_{P} \Lambda_{2}(0, P) \delta D(P) \\
& =\frac{1}{2} \int_{P}\left\{\Gamma_{2}(0, P)+\frac{1}{2} \int_{Q} \Gamma_{2}(0, Q) D_{2}^{2}(Q) \Lambda_{2}(Q, P)\right\} \delta D(P) \\
& =\frac{1}{2} \int_{P} \Gamma_{2}(0, P)\left[\delta D(P)+D_{2}^{2}(P) \tilde{\Pi}_{0}(P)\right] \\
& =\frac{1}{2} \int_{P} \Gamma_{2}(0, P)\left[D_{r}(P)+D_{2}^{2}(P)\left(\tilde{\Pi}_{0}(P)-\Pi_{0}(P)\right)\right]
\end{aligned}
$$

For $\Pi$ solution of the gap equation, we can use Eq. (IV.32) to replace $\tilde{\Pi}_{0}$ by $\Pi-\tilde{\Pi}_{2}-\tilde{\Pi}_{r}$ in the r.h.s. of this equation, and verify that the divergent terms linear in $\Pi_{0}\left(=\Pi-\Pi_{2}\right)$ cancel, as anticipated. We are left with:

$$
\begin{aligned}
\tilde{\Pi}_{0}(0) & =\frac{1}{2} \int_{P} \Gamma_{2}(0, P)\left\{D_{r}(P)-\tilde{\Pi}_{r}(P) D_{2}^{2}(P)\right\} \\
& +\frac{1}{2} \int_{P} \Gamma_{2}(0, P)\left\{\Pi_{2}(P)-\tilde{\Pi}_{2}(P)\right\} D_{2}^{2}(P) .
\end{aligned}
$$

The first line is finite, as it can be easily checked by using $D_{r}(P) \sim 1 / P^{6}, \tilde{\Pi}_{r}(P) \sim 1 / P^{2}$, and the fact that $\Gamma_{2}(0, P)$ behaves like $\ln P^{2}$. 
The remaining divergences in $\Pi(0)$ are therefore those of the following expression (we add $\left.\tilde{\Pi}_{2}(0)=0\right)$ :

$$
\frac{1}{2} \int_{P} \Gamma_{2}(0, P)\left\{\Pi_{2}(P)-\tilde{\Pi}_{2}(P)\right\} D_{2}^{2}(P)+\tilde{\Pi}_{2}(0)
$$

We verify now that the counterterm $\delta m^{2}$ absorbs the potential divergence. To do so,

observe first that this expression vanishes in the limit $m \rightarrow 0$ (then $\tilde{\Pi}_{2}(P)=\Pi_{2}(P)$ and $\left.\Pi_{2}(0)=0\right)$. Let us then expand $\Pi_{2}-\tilde{\Pi}_{2}=\Pi\left[D_{20}\right]-\Pi\left[D_{2}\right]$ around $D_{2}$ by using :

$$
D_{20}-D_{2}=\frac{1}{P^{2}+\Pi_{2}(P)}-\frac{1}{P^{2}+m^{2}+\Pi_{2}(P)}=m^{2} D_{2}^{2}(P)+D_{s}(P),
$$

where $D_{s}(P) \sim 1 / P^{6}$ at large $P$. We get

$$
\Pi_{2}(P)-\tilde{\Pi}_{2}(P)=-\delta m^{2}+\frac{1}{2} m^{2} \int_{Q} \Lambda_{2}(P, Q) D_{2}^{2}(Q)+\Pi_{s}(P),
$$

where $\Pi_{s}(P)$ is finite and goes as $1 / P^{2}$ at large $P$. Inserting this in Eq. (IV.36), and keeping only the terms which are not manifestly finite, we obtain:

$$
\begin{aligned}
& \frac{m^{2}}{2} \int_{Q}\left\{\frac{1}{2} \int_{P} \Gamma_{2}(0, P) D_{2}^{2}(P) \Lambda_{2}(P, Q)-\Lambda_{2}(0, Q)\right\} D_{2}^{2}(Q) \\
& +\delta m^{2}\left\{1-\frac{1}{2} \int_{P} \Gamma_{2}(0, P) D_{2}^{2}(P)\right\}
\end{aligned}
$$

One recognizes in this expression the right hand side of Eq. (IV.18) which specifies the value of the mass counterterm. It is therefore finite.

\section{E. Finite temperature}

It is straightforward to extend the diagrammatic analysis presented earlier in this section to the case of finite temperature. Doing so, one easily verifies that, iteration by iteration, all contributions involving the temperature are finite. Potential divergences coming from counterterms multiplying finite temperature contributions cancel out when subdivergences are eliminated. Furthermore, since the counterterms depend only on the 
asymptotic part of the propagator, which is unaffected by finite temperature effects, they are manifestly independent of the temperature. (The fact that $\Pi_{2}(P)$ is not modified by the temperature follows from Weinberg's theorem [15, 24], which ensures that the asymptotic behaviour $\sim P^{2} F\left(\ln P^{2}\right)$ of the self-energy comes from vacuum diagrams in which the internal momenta in all the internal lines are large and proportional to $P$.)

But although this diagrammatic analysis reveals that no difficulty of principle should be expected, in actual calculations the temperature dependent contributions may become mixed in a subtle way with vacuum, divergent contributions, and some work may be required to disentangle them. To be specific, we consider here the calculation in the imaginary time formalism. It is possible to organize the summation over the Matsubara frequencies in such a way as to separate the explicit thermal dependence in terms of integrals with 0,1 and 2 thermal factors:

$$
\Pi(K)=\Pi_{0 \mathrm{n}}(K)+\Pi_{1 \mathrm{n}}(K)+\Pi_{2 \mathrm{n}}(K) .
$$

The first contribution, $\Pi_{0 \mathrm{n}}(K)$ is exactly the same functional of $D$ as at zero temperature, Eq. (IV.2). Since $D$ depends on the temperature (because $\Pi$ does), $\Pi_{0 \mathrm{n}}(K)$ depends implicitly on the temperature. The term with one statistical factor, $\Pi_{1 \mathrm{n}}(K)$, can be written as:

$$
\Pi_{1 \mathrm{n}}(K)=\frac{1}{2} \int_{\tilde{P}} \sigma(\tilde{P}) \Lambda(K, \tilde{P})
$$

where $\Lambda$ is the same functional of $D$, Eq. (IV.3), as at zero temperature and depends only implicitly on the temperature through $D$. We have set $\sigma(\tilde{P})=\rho\left(p_{0}, p\right) n_{\left|p_{0}\right|} \varepsilon_{p_{0}}$, where $\rho\left(p_{0}, p\right)$ is the spectral function for the propagator $D$ :

$$
D(\omega, p)=\int_{-\infty}^{\infty} \frac{\mathrm{d} p_{0}}{2 \pi} \frac{\rho\left(p_{0}, p\right)}{p_{0}-\omega}
$$

$n_{p}=1 /\left(\mathrm{e}^{p / T}-1\right)$, and $\varepsilon_{p_{0}}= \pm 1$ depending on the sign of $p_{0}$. Finally, we have introduced the notation $\tilde{P}=\left(p_{0}, p\right)$, to be distinguished from $P=\left(i p_{0}, p\right)$. The 4-point functions such as $\tilde{\Lambda}_{2}(K, \tilde{P})$ are analytically continued in the complex plane from the complex value $i p_{0}$ where they are well defined (note that since $\Lambda(K, P)$ is a function of $K+P$, it remains 
well defined when $P$ is continued to $\tilde{P}$ ). Finally the term with two statistical factors, $\Pi_{2 \mathrm{n}}(K)$, can be written as:

$$
\Pi_{2 \mathrm{n}}(K)=\frac{\lambda^{2}}{2} \int_{\tilde{P}} \int_{\tilde{Q}} \sigma(\tilde{P}) \sigma(\tilde{Q}) D(K-\tilde{P}-\tilde{Q}) .
$$

As in the previous subsection, we write $\Pi=\Pi_{2}+\Pi_{0}$, with $\Pi_{2}$ determined by the zero-temperature auxiliary gap equation (IV.26) , and $\Pi_{0}(K)$ is at most logarithmic and carries the whole dependence upon $T$. For $\Pi_{0 \mathrm{n}}(K)$ we set again:

$$
\Pi_{0 \mathrm{n}}(K)=\tilde{\Pi}_{2}(K)+\tilde{\Pi}_{0}(K)+\tilde{\Pi}_{r}(K)
$$

where $\tilde{\Pi}_{2}, \tilde{\Pi}_{0}$, and $\tilde{\Pi}_{r}$ are given by the same Euclidean integrals as in the previous subsection, but contain implicit temperature dependence through the propagator $D$, except for $\tilde{\Pi}_{2}$ which depends only on $D_{2}$ and remains therefore temperature independent. It is also convenient to write

$$
\Pi_{1 \mathrm{n}}(K)=\tilde{\Pi}_{1}(K)+\tilde{\Pi}_{3}(K)
$$

where

$$
\tilde{\Pi}_{1}(K)=\frac{1}{2} \int_{\tilde{P}} \sigma(\tilde{P}) \Lambda_{2}(K, \tilde{P})
$$

is at most logarithmic at large $K$, and can therefore be considered as a modification of $\tilde{\Pi}_{0}$. As for $\tilde{\Pi}_{3}$, it decreases faster than a logarithm and it will be considered as a modification of $\tilde{\Pi}_{r}$. The same remark applies to $\Pi_{2 \mathrm{n}}(K)$ that we shall denote by $\tilde{\Pi}_{4}(K)$.

The final expression for the gap equation takes then a form similar to that at zero temperature, and may be written as

$$
\Pi=\tilde{\Pi}_{2}+\left(\tilde{\Pi}_{0}+\tilde{\Pi}_{1}\right)+\left(\tilde{\Pi}_{r}+\tilde{\Pi}_{3}+\tilde{\Pi}_{4}\right)=\tilde{\Pi}_{2}+\tilde{\Pi}_{0}^{\prime}+\tilde{\Pi}_{r}^{\prime}
$$

with $\tilde{\Pi}_{0}^{\prime}=\tilde{\Pi}_{0}+\tilde{\Pi}_{1}$ and $\tilde{\Pi}_{r}^{\prime}=\tilde{\Pi}_{r}+\tilde{\Pi}_{3}+\tilde{\Pi}_{4}$. Applying the strategy of the previous subsection to eliminate the vertex subdivergences in $\tilde{\Pi}_{0}^{\prime}(0)$, one gets easily:

$$
\begin{aligned}
\tilde{\Pi}_{0}^{\prime}(0) & =\frac{1}{2} \int_{P} D_{r}(P) \Gamma_{2}(0, P)+\frac{1}{2} \int_{\tilde{P}} \sigma(\tilde{P}) \Gamma_{2}(0, \tilde{P}) \\
& -\frac{1}{2} \int_{P} \Gamma_{2}(0, P) D_{2}^{2}(P)\left\{\Pi_{2}(P)-\tilde{\Pi}_{2}(P)-\tilde{\Pi}_{r}^{\prime}(P)\right\} .
\end{aligned}
$$


This equation generalize Eq. (IV.35). Its first line is finite. The divergences in the second line are temperature independent. When combined with those of $\tilde{\Pi}_{2}(0)$, they become identical to those of the zero temperature case, and are absorbed in $\delta m^{2}$.

\section{F. Massless case}

The massless case is interesting only at non-zero temperature. (Indeed, if $T=0$, then the gap equation with $m=0$ is simply the auxiliary gap equation that we have introduced before, cf. Eq. (IV.26), and whose solution is $\Pi_{2}$.) Consider then the finitetemperature case, where $\Pi$ admits the decomposition shown in Eq. (IV.40). When $m=0$, the subsequent manipulations of the term with zero thermal factors, $\Pi_{0 \mathrm{n}}$, deserve a special treatement: Indeed, if one proceeds as before, i.e., by developing $D$ around $D_{2}$ as in Eq. (IV.30), one meets infrared problems: since $D_{2}$ is now a massless propagator (this is the same as $D_{20}$ introduced before), the Bethe-Salpeter equation for the 4-point function $\Gamma_{2}$ is infrared divergent, and so are also $\tilde{\Pi}_{r}$ and $D_{r}$ used in (IV.35). One can however follow the same strategy as in Sec. IIIE and exploit the fact that the finite temperature effects generate a natural infrared cutoff. Since the ultraviolet divergences are ultimately sensitive only to the asymptotic behaviour of the propagator, they will not be affected by the replacement of $D_{2}$ by $D$ at appropiate places in the previous expansions. Specifically, we shall exploit the fact that $\Pi_{2}$ is the solution to the massless gap equation at $T=0$, namely (cf. Eq. (IV.26) $): \Pi_{2}=\Pi_{0 \mathrm{n}}\left[D_{2}\right]$. Then, after rewriting the full propagator as in Eq. (III.64):

$$
D_{2}(P)=D(P)+D(P) \Pi_{0}(P) D(P)-D_{r}^{\prime}(P) \equiv D-\delta D^{\prime},
$$

one can develop $\Pi_{0 \mathrm{n}}\left[D_{2}\right]$ around $\Pi_{0 \mathrm{n}}[D]$ without encountering infrared divergences:

$$
\Pi_{0 \mathrm{n}}\left[D_{2}\right]=\Pi_{0 \mathrm{n}}[D]-\frac{1}{2} \int_{P} \Lambda(K, P) \delta D^{\prime}(P)-\tilde{\Pi}_{r}^{\prime}(K),
$$

or, equivalently (compare to Eq. (IV.44)):

$$
\Pi_{0 \mathrm{n}}(K)=\Pi_{2}(K)+\frac{1}{2} \int_{P} \Lambda(K, P) \delta D^{\prime}(P)+\tilde{\Pi}_{r}^{\prime}(K),
$$


where $\Pi_{0 \mathrm{n}} \equiv \Pi_{0 \mathrm{n}}[D]$. Note that, as compared to Eq. (IV.31), the development above involves the kernel $\Lambda$ built with the full propagator, and similarly for $\delta D^{\prime}$. It is then possible to use the complete Bethe-Salpeter equation (with propagator $D$ ) in order to replace $\Lambda$ in terms of the infrared finite 4-point function $\Gamma$.

The subsequent manipulations proceed as before, in Sect. IVE except for the replacements $\Gamma_{2} \rightarrow \Gamma$ and $\delta D \rightarrow \delta D^{\prime}$. Since $\Pi_{2} \equiv \tilde{\Pi}_{2}$ when $m=0$, this finally leads to the following, manifestly finite, expression for $\tilde{\Pi}_{0}^{\prime}$ (compare to Eq. (IV.48) $)$ :

$$
\tilde{\Pi}_{0}^{\prime}(K)=\frac{1}{2} \int_{P} \Gamma(K, P)\left[D_{r}^{\prime}(P)-\tilde{\Pi}_{r}^{\prime}(P) D^{2}(P)\right]+\frac{1}{2} \int_{\tilde{P}} \sigma(\tilde{P}) \Gamma(K, \tilde{P}) .
$$

Since, however, the Bethe-Salpeter equation for $\Gamma$ involves the full propagator $D$, and thus is sensitive to the complete self-energy $\Pi=\Pi_{2}+\tilde{\Pi}_{0}+\tilde{\Pi}_{r}^{\prime}$, it follows that, unlike in the massive case, the Bethe-Salpeter and the (full) gap equation are now coupled, and must be solved simultaneously.

\section{GENERALIZATION TO HIGHER-LOOP ORDER}

In order to generalize the previous considerations to higher-loop order skeletons, we note first that many of the results that have been obtained in the previous section are independent of the precise structure of the skeleton considered. In particular, the equations which determine the vertex and mass counterterms, i.e. the Bethe-Salpeter equation (II.34) and the equation (IV.18) for $\partial \Pi / \partial m^{2}$, have been used in the previous section in their general forms, without further specifying the diagrammatic structure of $\Phi$, $\Pi$, or $\Lambda$. Similarly, the manipulations performed on these equations, like the expansion of the gap equation in Eq. (IV.32), or the expression (IV.31) for $\tilde{\Pi}_{0}(K)$, are generic as well, and would hold for any choice of the skeletons in $\Phi[D]$. There are new features however that need to be discussed. They are already present in the 4-loop approximation to $\Phi[D]$, and we shall illustrate our discussion with this example. The corresponding diagrams are displayed in Fig. 22

As we have seen in the discussion of the 3-loop approximation in Sect. IV, skeleton diagrams with different number of loops get mixed under renormalization: the two-loop 


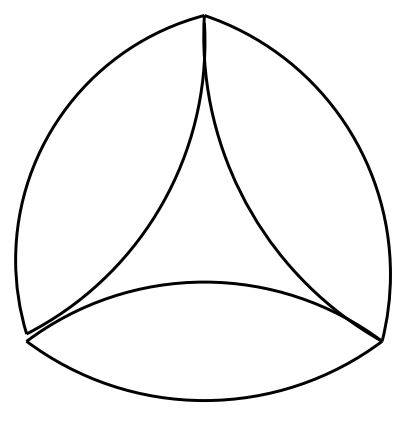

(a)

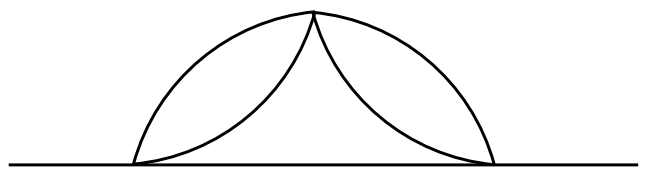

(b)

FIG. 22: The 4-loop contribution to $\Phi$ (a) and the corresponding 3-loop contribution to П (b).

skeleton is indeed needed as a counterterm to eliminate vertex subdivergences of the threeloop skeleton. We shall call primary skeletons the skeletons which are selected in the $\Phi$-derivable approximation that one considers, and counterterm skeletons those skeletons which carry the counterterms needed to remove the vertex subdivergences of the primary skeletons. To be specific, let us order the skeleton diagams according to their number of loops, and consider the approximation in which the primary skeletons are $(N+1)$-loop diagrams (so that the corresponding diagrams for $\Pi$ involve $N$ loops). The removal of divergences in the gap equation for $\Pi$ proceeds in three steps:

i) In the first step, one determines the 4-point function $\Lambda$ from $\Pi$ by using Eq. (II.16), and one constructs the vertex counterterms which render $\Lambda$ finite. Since obtained by opening one line in $\Pi$, the 4 -point function $\Lambda$ is given by primary diagrams with $N-1$ loops. In general, these diagrams will involve vertex subdivergences. The corresponding counterterms are constructed as in ordinary perturbation theory, with however the propagator $D$ associated to the lines of the diagrams; that is, they are the divergent parts of the loop integrals associated to the respective (sub)graphs evaluated with propagator $D$. These vertex counterterms are to be inserted in self-energy diagrams with $L<N$ loops (the "countertem skeletons"), while the primary skeletons are calculated with the renormalized coupling at their vertices. 

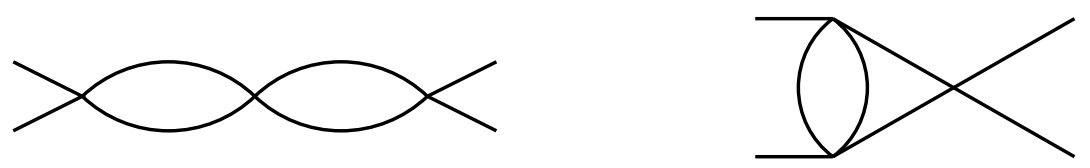

FIG. 23: The 2-loop contribution to $\Lambda$.

In the previous examples, we have met one counterterm of this type, namely the counterterm $\delta \lambda_{0}$ required to remove the global divergence of the one-loop contribution to $\Lambda$. For the 3-loop primary self-energy in Fig. 22. b, the corresponding, 2-loop, contributions to $\Lambda$ are displayed in Fig. 23, and their various divergences are exhibited in Fig. 24. These are both one-loop and 2-loop vertex counterterms, which are included in the vertices of the 2-loop and, respectively, one-loop self-energy graphs, as shown in Fig. 25. Note that the one-loop counterterms, which cancel subdivergences of $\Lambda$, involve all the possible channels. By contrast, the 2-loop counterterm, which is a global divergence of this particular contribution to $\Lambda$, involves only the $s$ and $u$ channels (so like $\delta \lambda_{0}$ in the example of Sect. IV). Clearly, this is related to the irreducibility of $\Lambda$ in one particular channel, the $t$-channel.
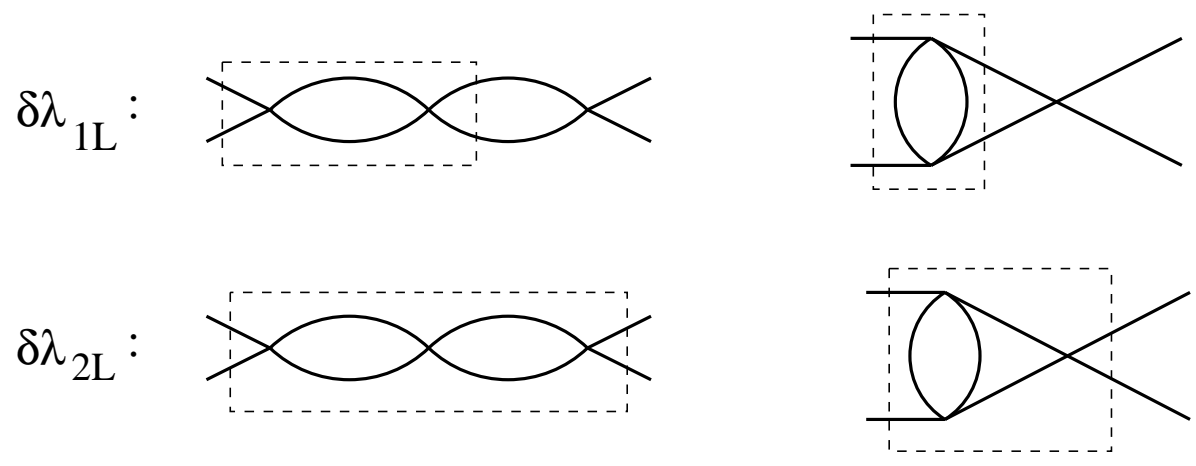

FIG. 24: The subgraphs enclosed in the dashed line boxes correspond to the divergences in $\Lambda$, and also to the vertex counterterms shown in Fig. 25. The contribution to $\delta \lambda_{1 \mathrm{~L}}$ represented by the diagram on the left corresponds to the $s$ and $u$ channels, while that on the right corresponds to the $t$ channel. 


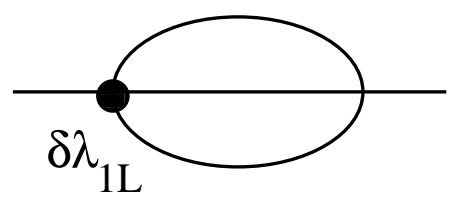

(a)

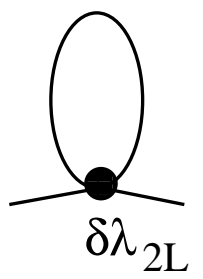

(b)

FIG. 25: The counterterm diagrams necessary to remove vertex subdivergences from the 3-loop self-energy in Fig. 22, b.

This example can be generalized to all orders: For a $N$-loop primary self-energy, the vertex counterterms associated with subdivergences in $\Lambda$ include all the possible channels, and appear in the vertices of self-energy skeletons with $2 \leq L<N-1$ loops (in such a way that $L+l=N$, where $l$ is the loop number of the vertex counterterm). As for the global divergence of $\Lambda$, this gives a $(N-1)$-loop counterterm which excludes all the topologies which are 2-particle-reducible in the $t$ channel. This counterterm is included in the one-loop self-energy diagram (the "tadpole").

Note that, as a result of this first step, the counterterms needed to renormalize $\Lambda$ are not explicitly computed, but rather expressed as integrals which involve the (yet unknown) propagator $D$. However, the divergent parts of these integrals are sensitive only to the leading high momentum behaviour of the propagator, i.e., to $D_{2}$. Similarly, $\tilde{\Lambda}$ (the sum of $\Lambda$ plus the vertex counterterms constructed at this step) is a finite functional of $D$, or $D_{2}$.

ii) The second step refers to the renormalization and the calculation of the asymptotically equivalent propagator $D_{2}$. To that aim, one proceeds as in the 3-loop example in Sec. IVC - that is, one considers the self-consistent gap equation with $m=0$ - except that, in the general case, one has to include in the r.h.s. of the equation for $\Pi$ all the counterterm diagrams determined in step $i$ ). Then, within dimensional regularisation, the functional $\Pi\left[D_{2}\right]$ (the r.h.s. of the gap equation) has neither vertex, nor mass, divergences, but only a global field divergence, proportional to $K^{2}$, that can be absorbed in 
the counterterm $\delta Z K^{2}$. After this divergence is subtracted, the ensuing, finite, equation, can be solved for $\Pi_{2}$. This gives the propagator $D_{2}$, which then can be used to explicitly compute $\tilde{\Lambda}_{2}$.

iii) In the third step, the (finite) kernel $\tilde{\Lambda}_{2}$ together with the propagator $D_{2}$ are used to construct the Bethe-Salpeter equation, together with the equation for $\delta \Pi / \delta m^{2}$. By renormalizing these equations, we obtain the countertermes $\delta \lambda$ and $\delta m^{2}$ required to eliminate the residual vertex subdivergences and, respectively, the mass divergences from the gap equation. Note that, in contrast to the vertex counterterms for $\Lambda$ (cf. the first step), which involve, at most, $N-1$ loops, those obtained by iterating the Bethe-Salpeter equation will receive contributions with arbitrarily many loops (and similarly for $\delta m^{2}$ ). At this stage, one can use the algebraic derivation in Sect. IV.E to verify that the gap equation is a finite equation for $\Pi_{0} \equiv \Pi-\Pi_{2}$.

The arguments in Sect. IV.E rely on two properties which remain true in any order in the skeleton expansion, as we shall see. These properties are:

A) $\Lambda(P, K)-\Lambda(P, 0)$, considered as a function of $P$ for fixed $K$, is of degree of divergence less than zero.

B) The term $\tilde{\Pi}_{r}(K)$ in the r.h.s. of Eq. (IV.32) is finite and decreases like $1 / K^{2}$ at large $K$.

The first property, on the behaviour of $\Lambda(P, K)-\Lambda(P, 0)$ at large $P$ for fixed $K$, follows from the 2-particle irreducibility of $\Lambda$, as we explain now. The different contributions to the logarithmic behaviour of the 4-point function at fixed $K$ and large $P$ are, according to Weinberg's theorem, associated to 4-point subgraphs attached to the two external lines carrying $P$. Recall that, in Weinberg's terminology, a subgraph is any set of lines choosen in the initial graph in such a way that there is no vertex attached to only one line of the subgraph [15, 24]. In Fig. 26, we give examples of 4-point subgraphs attached to $P$ for a graph contributing to $\Gamma(P, K)$. We also give an example of a set of lines which is not a subgraph. For both $\Gamma(P, K)$ and $\Lambda(P, K)$, there is always a trivial four point subgraph attached to $P$ : the graph itself. Clearly the leading behaviour at large $P$ for this contribution is independent of $K$, either for $\Gamma(P, K)$ or $\Lambda(P, K)$. The reason is 

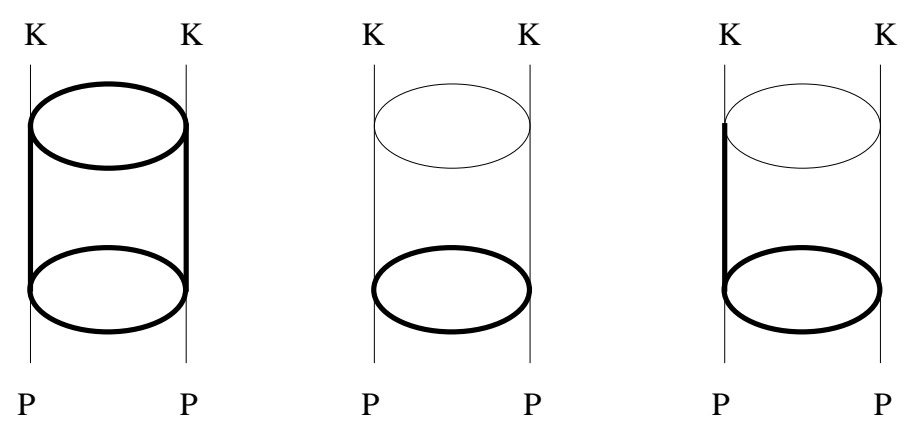

FIG. 26: Examples and counter-example of 4-point subgraphs attached to $P$. (a) and (b) are 4-point subgraphs contributing to the dominant behaviour of $\Gamma(P, K)$ at large $P$ and fixed $K$. This leading contribution is independent of $K$ in the case of (a) but the subgraph (b) may give a $K$-dependent contribution. (c) is not a subgraph.

simply that for this subgraph, $K$ appears in propagators where, by asumption, the total momenta goes to infinity, while $K$ is kept finite. Of course there are other logarithmic contributions. The second diagram in Fig. 26 gives an example of such a contribution in which the leading contribution at large $P$ does depend on $K$. But it is easily verified that this particular 2-particle reducible topology is the only one allowing for a $K$-dependence. Since $\Lambda(P, K)$ is irreducible, its asymptotic behaviour at large $P$ is independent of $K$. Property A) then follows.

For the second property, we note that $\tilde{\Pi}_{r}$ is obtained by differentiating twice a skeleton graph for the self-energy. Thus $\tilde{\Pi}_{r}$ has the structure of a 6-point function which is closed into a two-point function by two $\delta D$, each of degree of divergence -4 . The internal sixpoint subgraph itself has degree of divergence -2 , and since all (vertex) subdivergences supposed to be eliminated, this is strictly finite. When the two effective propagators $\delta D$ are closed on it, the UV finiteness is preserved because one of the two following situations occurs: 1) The two propagators appear together in the same loop; that loop is certainly finite. 2) If a single effective propagator appear in some loop, that loop is certainly not a tadpole (since the whole graph has the topology of a skeleton), and thus contains at least one additional propagator belongning to the six-point vertex subgraph; that loop is finite too. Since the overall graph is finite, simple power counting together with Weinberg's 
theorem ensures that, at large $K, \tilde{\Pi}_{r}(K) \sim 1 / K^{2}$.

To conclude, let us briefly discuss the scale dependence of the renormalized coupling constant which emerges from the renormalization program described above. (A similar discussion applies to the $\mu$-dependence of $\Pi_{2}$ and of the renormalized mass parameter $m$.) Consider the $N$-loop approximation to the self-consistent self-energy which includes all the primary skeletons with $L \leq N$ loops, together with the corresponding counterterm skeletons. Given the similitude between step $i$ ) above and ordinary perturbation theory, it is clear that renormalized vertices belonging to diagrams with different numbers of loops are dressed differently, and thus run differently with $\mu$. The respective $\mu$-dependences are non-perturbative, since contributions of all orders in $\lambda$ are included (because the corresponding counterterms depend upon $\Pi_{2}$, and also because of the iterations performed by the Bethe-Salpeter equation in the case of the coupling constant which enters the tadpole). However, when restricted to an expansion in powers of $\lambda$, the $\mu$-dependences of the vertices are those expected from the loop order of the included diagrams. In particular, the couplings in the vertices of the $N$-loop primary diagrams are not dressed at all, so they are independent of $\mu$, those which enter the $(N-1)$-loop primary diagrams are running according to the one-loop beta function, etc. The coupling constant in the one-loop selfenergy is exceptional, in that it exhibits the most developped running (perturbatively correct up to loop order $N-1$ ).

The one-loop example discussed in Sect. III (see, especially, Sect. III.D) suggests that it should be possible to exploit the non-perturbative character of the $\mu$-dependence of the vertices in order to minimize the scale dependence of the self-consistent solutions for $\Gamma$ and $\Pi$. In that one-loop case, we have in fact been able to verify explicitely that both $\Gamma$ and $\Pi$ are strictly independent of $\mu$. In the general case, the corresponding analysis is considerably more involved, and will not be pursued here. We shall only emphasize a specific feature of the $\Phi$-derivable approximations, which is that vertices in different diagrams run differently with $\mu$. This should be taken into account when trying to reduce (and possibly eliminate) the scale dependence of the physical observables. 
[1] J. M. Luttinger and J. C. Ward, Phys. Rev. 118, 1417 (1960).

[2] G. Baym and L. Kadanoff, Phys. Rev. 12722 (1962); G. Baym, Phys. Rev. 127, 1391 (1962).

[3] C. De Dominicis and P. C. Martin, J. Math. Phys. 5, 14, 31 (1964).

[4] J. M. Cornwall, R. Jackiw and E. Tomboulis, Phys. Rev. D 10, 2428 (1974).

[5] J. P. Blaizot, E. Iancu and A. Rebhan, Phys. Rev. Lett. 83, 2906 (1999); Phys. Lett. B470, 181 (1999); Phys. Rev. D 63, 065003 (2001).

[6] B. Vanderheyden and G. Baym, J. Stat. Phys. 93, 843 (1998).

[7] A. Peshier, Phys. Rev. D63, 105004 (2001).

[8] E. Braaten and E. Petitgirard, Phys. Rev. D65, 041701 (2002); Phys. Rev. D65, 085039 (2002).

[9] F. Karsch, A. Patkós and P. Petreczky, Phys. Lett. B401, 69 (1997).

[10] J. Berges, Nucl. Phys. A699, 847 (2002); G. Aarts, D. Ahrensmeier, R. Baier, J. Berges and J. Serreau, Phys. Rev. D 66, 045008 (2002).

[11] H. van Hees and J. Knoll, Phys. Rev. D 66, 025028 (2002)

[12] A. Arrizabalaga and J. Smit, Phys. Rev. D 66, 065014 (2002)

[13] L. Dolan and R. Jackiw, Phys. Rev. D 9, 3320 (1974).

[14] I. T. Drummond, R. R. Horgan, P. V. Landshoff and A. Rebhan, Nucl. Phys. B 524, 579 (1998)

[15] J. C. Collins, "Renormalization. An Introduction To Renormalization, The Renormalization Group, And The Operator Product Expansion," Cambridge, Uk: Univ. Pr. ( 1984) 380p

[16] J. P. Blaizot, R. Mendez-Galain and N. Wschebor, Annals Phys. 307, 209 (2003).

[17] H. van Hees and J. Knoll, Phys. Rev. D 65, 025010 (2002)

[18] H. Van Hees and J. Knoll, Phys. Rev. D 65, 105005 (2002)

[19] N.N. Bogoliubov and O.S. Parasiuk, Acta Math. 97, 227 (1957); K. Hepp, Commun. Math. Phys. 2, 301 (1966). 
[20] J. P. Blaizot, E. Iancu and U. Reinosa, Phys. Lett. B 568, 160 (2003).

[21] J. I. Kapusta, Finite-temperature field theory (Cambridge University Press, Cambridge, 1989).

[22] M. Le Bellac, Thermal Field Theory (Cambridge University Press, Cambridge, 1996).

[23] J.P. Blaizot and G. Ripka, Quantum Theory of Finite Systems (MIT Press, Cambridge, 1986).

[24] S. Weinberg, Phys. Rev. 118, 838 (1960).

[25] P. Ramond, Field Theory: A Modern Primer (Addison-Wesley, 1989). 